\title{
PASSAGE THROUGH A POTENTIAL BARRIER AND MULTIPLE WELLS
}

\author{
D. R. YAFAEV
}

To the memory of Vladimir Savel'evich Buslaev

\begin{abstract}
Consider the semiclassical limit, as the Planck constant $\hbar \rightarrow 0$, of bound states of a one-dimensional quantum particle in multiple potential wells separated by barriers. We show that, for each eigenvalue of the Schrödinger operator, the Bohr-Sommerfeld quantization condition is satisfied at least for one potential well. The proof of this result relies on a study of real wave functions in a neighborhood of a potential barrier. We show that, at least from one side, the barrier fixes the phase of wave functions in the same way as a potential barrier of infinite width. On the other hand, it turns out that for each well there exists an eigenvalue in a small neighborhood of every point satisfying the Bohr-Sommerfeld condition.
\end{abstract}

\section{INTRODUCTION}

1.1. Our goal is to study the semiclassical limit as $\hbar \rightarrow 0$ of eigenvalues $\lambda=\lambda(\hbar)$ of the one-dimensional Schrödinger equation

$$
-\hbar^{2} \psi^{\prime \prime}(x ; \lambda, \hbar)+v(x) \psi(x ; \lambda, \hbar)=\lambda(\hbar) \psi(x ; \lambda, \hbar), \quad v=\bar{v}, \quad \psi=\bar{\psi} \in L^{2}(\mathbb{R}),
$$

for the case of several potential wells $X_{1}(\lambda), \ldots, X_{L}(\lambda)$ where $v(x)<\lambda$ separated by barriers $B_{1}(\lambda), \ldots, B_{L-1}(\lambda)$ where $v(x)>\lambda$. We suppose that the energy $\lambda$ is non-critical; in particular, it is separated from bottoms of the wells and tops of the barriers. We also assume that $v(x) \geq v_{0}$ for some $v_{0}>\lambda$ and large $|x|$, that is, there are infinite barriers to the left of $X_{1}(\lambda)$ and to the right of $X_{L}(\lambda)$. Of course the spectrum of the Schrödinger operator $H(\hbar)=-\hbar^{2} d^{2} / d x^{2}+v(x)$ below the point $v_{0}$ is discrete. It is a common wisdom that the limit of its eigenvalues $\lambda(\hbar)$ as $\hbar \rightarrow 0$ is described by some version of the Bohr-Sommerfeld quantization condition. However in the case of multiple wells, we have not found a precise formulation and a proof of this statement in the literature. So we are aiming at filling in this gap. Although quite elementary, some of the results obtained seem to be rather unexpected.

To explain them at a heuristic level, let us first recall briefly the thoroughly studied case of one potential well $X=X(\lambda)=\left(x_{-}, x_{+}\right)$where $x_{ \pm}=x_{ \pm}(\lambda)$ are the (only) solutions of the equation $v(x)=\lambda$. Put

$$
\Phi_{ \pm}(x ; \lambda)= \pm \int_{x}^{x_{ \pm}(\lambda)}(\lambda-v(y))^{1 / 2} d y, \quad x \in\left(x_{-}(\lambda), x_{+}(\lambda)\right)
$$

It follows from the Green-Liouville approximation (see, for example, the book [21, Chapter 6) that for $x \in X$ (inside the well) an arbitrary real solution of equation (1.1) has the asymptotics

$$
\psi(x ; \hbar)=A_{+}(\hbar)(\lambda-v(x))^{-1 / 4}\left(\sin \left(\hbar^{-1} \Phi_{+}(x)+\varphi_{+}(\hbar)\right)+O(\hbar)\right)
$$

and

$$
\psi(x ; \hbar)=A_{-}(\hbar)(\lambda-v(x))^{-1 / 4}\left(\sin \left(\hbar^{-1} \Phi_{-}(x)+\varphi_{-}(\hbar)\right)+O(\hbar)\right)
$$

2000 Mathematics Subject Classification. 47A40, 81U05.

Key words and phrases. Schrödinger equation, multiple potential wells, Bohr-Sommerfeld quantization conditions, fixing conditions. 
as $\hbar \rightarrow 0$. Here the amplitudes $A_{ \pm}(\hbar)=A_{ \pm}(\lambda, \hbar) \in \mathbb{R}_{+}$and the phases $\varphi_{ \pm}(\hbar)=\varphi_{ \pm}(\lambda, \hbar) \in$ $\mathbb{R} /(2 \pi \mathbb{Z})$. We emphasize that the phases are always defined up to terms of order $O(\hbar)$. H. Jeffreys [13] and H. Kramers [15] (see also the book [17]) observed that if $\psi \in L^{2}\left(\mathbb{R}_{ \pm}\right.$), then necessarily $\varphi_{ \pm}(\hbar)=\pi / 4(\bmod \pi)$. Their arguments are based on contouring the turning points $x_{ \pm}$in the complex plane.

Comparing relations (1.3) and (1.4) where $\varphi_{ \pm}(\hbar)=\pi / 4(\bmod \pi)$, one sees that if $\lambda$ is an eigenvalue of the operator $H(\hbar)$, then necessarily

$$
\Phi(\lambda):=\int_{x_{-}(\lambda)}^{x_{+}(\lambda)}(\lambda-v(y))^{1 / 2} d y=\pi(n+1 / 2) \hbar+O\left(\hbar^{2}\right), \quad \hbar \rightarrow 0,
$$

for some integer number $n=n(\lambda, \hbar)$. Formula (1.5) is known as the Bohr-Sommerfeld quantization condition. It shows that all eigenvalues of the operator $H(\hbar)$ in a fixed (not depending on $\hbar$ ) vicinity of a non-critical energy $\lambda$ cannot lie away of $O\left(\hbar^{2}\right)$ neighborhoods of the points $\Phi^{-1}(\pi(n+$ $1 / 2) \hbar)$. It can be additionally shown that there is exactly one eigenvalue of $H(\hbar)$ in each of these neighborhoods.

From the point of view of differential equations, these results were discussed in [25]. Analytically, the paper [25] was based on a detailed study of the asymptotics of eigenfunctions $\psi(x ; \hbar)$ of the operator $H(\hbar)$ as $\hbar \rightarrow 0$. As is well known (see again the book [21, Chapter 11), their behavior in neighborhoods of the turning points $x_{+}$and $x_{-}$can be described in terms of the Airy functions. It is also possible (see, for example, the books [24, Chapter VIII, or 77, Chapter IV) to justify the method of Jeffreys-Kramers, but this requires the stringent assumption that the potential $v(x)$ is an analytic function.

1.2. In the case of multiple wells $X_{1}(\lambda), \ldots, X_{L}(\lambda)$, one can write $L$ quantization conditions:

$$
\Phi_{\ell}(\lambda):=\int_{X_{\ell}(\lambda)}(\lambda-v(y))^{1 / 2} d y=\pi(n+1 / 2) \hbar+O\left(\hbar^{2}\right), \quad \hbar \rightarrow 0, \quad \ell=1, \ldots, L,
$$

where $n=n_{\ell}(\lambda, \hbar)$ are some integer numbers. We again consider all eigenvalues of the Schrödinger operator $H(\hbar)=-\hbar^{2} d^{2} / d x^{2}+v(x)$ in a vicinity of some non-critical energy $\lambda$ (so we avoid bottoms of wells and tops of barriers) and show that at least one of the quantization conditions (1.6) is satisfied for each eigenvalue.

The proof of this result relies on a study of real solutions $\psi(x ; \hbar)$ of equation (1.1) for an arbitrary non-critical $\lambda$ in a neighborhood of a potential barrier between some points $\left(b_{1}, b_{2}\right)$; thus $v\left(b_{j}\right)=\lambda$, $v(x)>\lambda$ for $x \in\left(b_{1}, b_{2}\right)$ and $v(x)<\lambda$ for $x \notin\left(b_{1}, b_{2}\right)$. Of course $b_{1}$ and $b_{2}$ depend on $\lambda$. Set

$$
\Theta_{j}(x, \lambda)=(-1)^{j} \int_{b_{j}(\lambda)}^{x}(\lambda-v(y))^{1 / 2} d y, \quad j=1,2 .
$$

The Green-Liouville approximation shows that solutions $\psi(x ; \hbar)$ are oscillating functions away from the barrier:

$$
\psi(x ; \hbar)=A_{1}(\hbar)(\lambda-v(x))^{-1 / 4}\left(\sin \left(\hbar^{-1} \Theta_{1}(x, \lambda)+\theta_{1}(\hbar)\right)+O(\hbar)\right)
$$

for $x<b_{1}$ and

$$
\psi(x ; \hbar)=A_{2}(\hbar)(\lambda-v(x))^{-1 / 4}\left(\sin \left(\hbar^{-1} \Theta_{2}(x, \lambda)+\theta_{2}(\hbar)\right)+O(\hbar)\right)
$$

for $x>b_{2}$ where the amplitudes $A_{j}(\hbar)=A_{j}(\lambda, \hbar)>0$ and the phases $\theta_{j}(\hbar)=\theta_{j}(\lambda, \hbar) \in \mathbb{R} /(2 \pi \mathbb{Z})$. The striking result is that $\theta_{j}(\hbar)=\pi / 4(\bmod \pi)$ for at least one $j=1,2$. So the phase $\theta_{j}(\hbar)$ is fixed by a finite barrier exactly in the same way as by an infinitely wide (and probably infinitely high) potential barrier (cf. formulas (1.3) and (1.4) for $\left.\varphi_{ \pm}(\hbar)=\pi / 4(\bmod \pi)\right)$. We use the term fixing condition for this fact. We emphasize that we consider real solutions of the Schrödinger equation. 
Thus our problem is different from the famous problem of tunneling of a wave through a potential barrier where the solutions considered are necessarily complex.

In the case of $L$ wells $X_{1}, \ldots, X_{L}$ separated by $L-1$ barriers, the stated result yields at least $L-1$ fixing conditions. Additionally, if $\lambda$ is an eigenvalue of the operator $H(\hbar)$, then according to formulas (1.3) and (1.4) where $\varphi_{ \pm}(\hbar)=\pi / 4(\bmod \pi)$ the fixing conditions hold at the left point of $X_{1}$ and at the right point of $X_{L}$. Thus, we have $L+1$ fixing conditions for $L$ wells, and hence there exists a well $X_{\ell}$ for which two fixing conditions are satisfied. The quantization condition (1.5) for this well can be obtained essentially in the same way as for the one-well problem.

Furthermore, we show that, for each $n$, there is at most one solution $\lambda$ of equation (1.6). Unfortunately, our proof of this fact requires an additional assumption. In general, this assumption is rather implicit, but it is automatically satisfied in the case of two symmetric wells.

Finally, constructing appropriate trial functions, we check that, for all $\ell=1, \ldots, L$ and $n \in \mathbb{Z}$ such that $\Phi_{\ell}^{-1}(\pi(n+1 / 2) \hbar)$ belongs to a neighborhood of $\lambda_{0}$, there is at least one eigenvalue of the operator $H(\hbar)$ in $O\left(\hbar^{2}\right)$ neighborhood of each point $\Phi_{\ell}^{-1}(\pi(n+1 / 2) \hbar)$. This yields a one-to-one correspondence between eigenvalues of the operator $H(\hbar)$ in a neighborhood of $\lambda_{0}$ and the points $\Phi_{\ell}^{-1}(\pi(n+1 / 2) \hbar)$.

1.3. This paper can be considered as continuation of [25] where the case of one potential well was studied in details. It is organized as follows. In Section 2 , we collect various results on asymptotics as $\hbar \rightarrow 0$ of solutions $\psi(x ; \hbar)$ of equation (1.1). It is a common wisdom that this asymptotics is described by the Green-Liouville approximation away from the turning points $x$ where $\lambda=v(x)$. In neighborhoods of the turning points the asymptotics of $\psi(x ; \hbar)$ is more complicated and is given in terms of Airy functions. The corresponding results were obtained by R. E. Langer in [18, 19] and thoroughly exposed, for example, by F. W. Olver in his book [21]. We essentially follow the scheme of [21]. The main difference concerns estimates of remainders. It is convenient to treat separately the classically allowed (where $v(x)<\lambda$ ) and forbidden (where $v(x)>\lambda$ ) regions which seems to be intuitively more clear. The results on solutions exponentially decaying in the classically forbidden region are borrowed from [25]; they are stated as Theorem 2.2. The results on solutions exponentially growing in the classically forbidden region are stated as Theorem 2.7. Its proof is postponed to Section 3.

The case of one potential well is considered in Section 4. Here our results are rather standard, but in contrast to usual presentations, we do not suppose that $\psi \in L^{2}(\mathbb{R})$ and our arguments are of a local nature.

The behavior of real solutions $\psi(x ; \hbar)$ of equation (1.1) in a neighborhood of a potential barrier is studied in Section 5. The main result where the fixing conditions are established is stated as Theorem 5.5. It is compared with tunneling of a wave (described by a complex solution of equation (1.1) ) through a barrier in Appendix.

Theorem [5.5]allows us to study the quantization conditions for eigenvalues in the case of multiple potential wells. This is done in Section 6; see Theorems 6.2, 6.3 and 6.4. Here we establish a oneto-one correspondence between eigenvalues of the Schrödinger operator $H(\hbar)=-\hbar^{2} d^{2} / d x^{2}+v(x)$ in a neighborhood of a non-critical energy and solutions of equations (1.6). The results of such type imply the semiclassical Weyl formula for the distribution of eigenvalues of the operator $H(\hbar)$ as $\hbar \rightarrow 0$ with a strong estimate of the remainder. It turns out (see Propositions 6.6 and 6.7) that this remainder never exceeds $L$.

1.4. The quantization conditions for one-dimensional multiple wells is of course one of basic problems in quantum mechanics. So it is rather surprising that it was thoroughly considered mainly for the case of two symmetric wells, and in the majority of the papers (see, e.g., 9, 10, 14, 20]) on this subject only the exponential splitting due to the tunneling through the barrier of two close eigenvalues was studied. A similar problem was also considered in the multi-dimensional case (see, 
e.g., [12, 23]). In several papers mentioned above, a special attention was paid to eigenvalues lying at bottoms of potential wells. There are also numerous papers (see, e.g., [8, [5]) where the BohrSommerfeld quantization condition was discussed near the tops of potential barriers. All these problems are quite far from the present article.

The closest to the present article is apparently the paper [11] by B. Helffer and D. Robert where the quantization conditions were studied for two symmetric potential wells. The paper [1] is written in a very general context of multidimensional pseudodifferential operators and, analytically, it relies on the semiclassical functional calculus combined with some results of the microlocal analysis (see the books [6, 22]). We emphasize that, for symmetric wells, there is only one quantization condition. It is stated in Theorem 3.9 of [1] (probably, there is a misprint in the formula of this theorem, the correct formula being given in Remark 3.13).

We finally note that semiclassical methods were successfully applied to essentially more difficult one-dimensional problems. For example, we mention the series of papers [2, 3, 4, by V. S. Buslaev with collaborators who developed semiclassical theory on a periodic background.

We emphasize that multi-dimensional problems are out of the scope of this article which relies exclusively on the methods of ordinary differential equations.

I thank Nicolas Raymond and San Vũ Ngoc for useful discussions of papers on microlocal analysis.

1.5. Let us introduce some standard notation. We denote by $C$ and $c$ different positive constants whose precise values are of no importance. The parameter $\hbar$ (the Planck constant) is always supposed to be sufficiently small. The phases in various asymptotic formulas such as (1.3), (1.4) or (1.8), (1.9) are defined up to terms of order $O(\hbar)$ as $\hbar \rightarrow 0$. All asymptotic relations are supposed to be differentiable with respect to $x$ and $\lambda$ with natural estimates of remainders in differentiated formulas.

We work in a neighborhood of some non-critical energy $\lambda_{0}$ (it means that $v^{\prime}(x) \neq 0$ if $v(x)=\lambda_{0}$ ). Various assumptions imposed at $\lambda_{0}$ are automatically satisfied for all $\lambda$ in some neighborhood $\left(\Lambda_{1}, \Lambda_{2}\right)$ of $\lambda_{0}$. We suppose of course that $\left(\Lambda_{1}, \Lambda_{2}\right)$ is separated from all critical values of $v(x)$. If it cannot lead to a confusion, the dependence of various objects on $\lambda$ is often neglected in the notation. The derivatives in $\lambda$ are usually denoted by dots.

The conditions on $v(x)$ at infinity are not very important; for example, $v(x)$ may tend to $+\infty$ or finite limits as $x \rightarrow \pm \infty$. However, we assume that

$$
\liminf _{x \rightarrow \pm \infty} v(x)>\lambda_{0}
$$

so that there are infinite barriers to the left of the well $X_{1}$ and to the right of the well $X_{L}$.

\section{Semiclassical solutions of the Schrödinger equation}

In this section we construct solutions of the equation (1.1) oscillating inside a potential well and (super-) exponentially decaying or growing inside a potential barrier. This standard construction will of course be given in terms of the Airy functions.

2.1. Let us recall the definition of Airy functions and their necessary properties (see, e.g., the book [21], Chapter 11.1, for details). Consider the equation

$$
-\theta^{\prime \prime}(t)+t \theta(t)=0
$$

and denote by $\operatorname{Ai}(t)$ its solution with asymptotics

$$
\operatorname{Ai}(t)=2^{-1} \pi^{-1 / 2} t^{-1 / 4} \exp \left(-2 t^{3 / 2} / 3\right)\left(1+O\left(t^{-3 / 2}\right)\right), \quad t \rightarrow+\infty .
$$

Then

$$
\operatorname{Ai}(t)=\pi^{-1 / 2}|t|^{-1 / 4} \sin \left(2|t|^{3 / 2} / 3+\pi / 4\right)+O\left(|t|^{-7 / 4}\right), \quad t \rightarrow-\infty .
$$


The solution $\operatorname{Bi}(t)$ of equation (2.1) is defined by its asymptotics as $t \rightarrow-\infty$ which differs from (2.3) only by the phase shift:

$$
\operatorname{Bi}(t)=\pi^{-1 / 2}|t|^{-1 / 4} \cos \left(2|t|^{3 / 2} / 3+\pi / 4\right)+O\left(|t|^{-7 / 4}\right), \quad t \rightarrow-\infty .
$$

It grows exponentially at $+\infty$ :

$$
\operatorname{Bi}(t)=\pi^{-1 / 2} t^{-1 / 4} \exp \left(2 t^{3 / 2} / 3\right)\left(1+O\left(t^{-3 / 2}\right)\right), \quad t \rightarrow+\infty .
$$

Note that $\operatorname{Ai}(t)>0$ and $\operatorname{Bi}(t)>0$ for all $t \geq 0$.

We also use that all asymptotics (2.2), (2.3) and (2.4), (2.5) can be differentiated in $t$. In this case the remainders $O\left(|t|^{-3 / 2}\right)$ in (2.2), (2.5) remain unchanged and $O\left(|t|^{-7 / 4}\right)$ in (2.3), (2.4) is replaced by $O\left(|t|^{-5 / 4}\right)$. In particular, the Wronskian

$$
\{\operatorname{Ai}(t), \operatorname{Bi}(t)\}:=\operatorname{Ai}^{\prime}(t) \operatorname{Bi}(t)-\operatorname{Ai}(t) \operatorname{Bi}^{\prime}(t)=-\pi^{-1} .
$$

2.2. For a point $x_{0} \in \mathbb{R}$ and the signs "+" or "-", we make the following

Assumption 2.1. The function $v \in C^{2}$ on some interval $\left(x_{1}, x_{2}\right)$ containing $x_{0}$ and $\pm(v(x)-$ $\left.v\left(x_{0}\right)\right)<0$ for $x \in\left(x_{1}, x_{0}\right)$ and $\pm\left(v(x)-v\left(x_{0}\right)\right)>0$ for $x \in\left(x_{0}, x_{2}\right)$. Moreover, the function $v$ belongs to the class $C^{3}$ in some neighborhood of the point $x_{0}$ and $\pm v^{\prime}\left(x_{0}\right)>0$.

Let $\lambda=v\left(x_{0}\right)$ in the equation (1.1). If Assumption 2.1 holds for the sign "+", then $\left(x_{1}, x_{0}\right)$ is a potential well and $\left(x_{0}, x_{2}\right)$ is a potential barrier. On the contrary, if it holds for the sign " - ", then $\left(x_{1}, x_{0}\right)$ is a potential barrier and $\left(x_{0}, x_{2}\right)$ is a potential well. In both cases $x_{0}$ is the turning point. Note that Assumption 2.1 is automatically satisfied for all $x_{0}^{\prime}$ in some neighborhood of $x_{0}$.

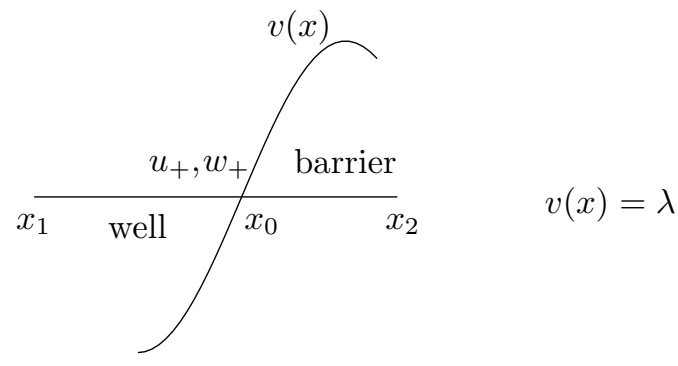

Case "+"

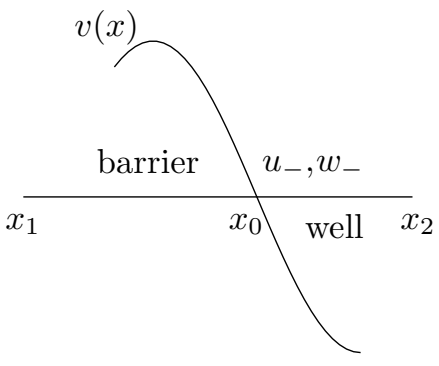

Case " - "

Our goal in this section is to describe asymptotics as $\hbar \rightarrow 0$ of solutions $u_{ \pm}\left(x ; x_{0}, \hbar\right)$ of the equation (1.1) exponentially decaying and of solutions $w_{ \pm}\left(x ; x_{0}, \hbar\right)$ exponentially growing inside the barrier. Both these solutions are oscillating inside the well.

To state results, we need the following auxiliary functions:

$$
\begin{aligned}
& \xi_{+}\left(x ; x_{0}\right)=\left(\frac{3}{2} \int_{x_{0}}^{x}\left(v(y)-v\left(x_{0}\right)\right)^{1 / 2} d y\right)^{2 / 3}, \quad x \in\left(x_{0}, x_{2}\right), \\
& \xi_{+}\left(x ; x_{0}\right)=-\left(\frac{3}{2} \int_{x}^{x_{0}}\left(v\left(x_{0}\right)-v(y)\right)^{1 / 2} d y\right)^{2 / 3}, \quad x \in\left(x_{1}, x_{0}\right),
\end{aligned}
$$

and

$$
\begin{aligned}
& \xi_{-}\left(x ; x_{0}\right)=\left(\frac{3}{2} \int_{x}^{x_{0}}\left(v(y)-v\left(x_{0}\right)\right)^{1 / 2} d y\right)^{2 / 3}, \quad x \in\left(x_{1}, x_{0}\right), \\
& \xi_{-}\left(x ; x_{0}\right)=-\left(\frac{3}{2} \int_{x_{0}}^{x}\left(v\left(x_{0}\right)-v(y)\right)^{1 / 2} d y\right)^{2 / 3}, \quad x \in\left(x_{0}, x_{2}\right) .
\end{aligned}
$$


D. R. YAFAEV

Since the point $x_{0}$ is fixed, for shortness, in this section we set

$$
\xi_{ \pm}(x)=\xi_{ \pm}\left(x ; x_{0}\right) \quad \text { and } \quad u_{ \pm}(x ; \hbar)=u_{ \pm}\left(x ; x_{0}, \hbar\right), \quad w_{ \pm}(x ; \hbar)=w_{ \pm}\left(x ; x_{0}, \hbar\right) .
$$

It is easy to show (see Lemma 3.1 in Chapter 11 of [21, for details) that $\xi_{ \pm} \in C^{3}\left(x_{1}, x_{2}\right)$, $\pm \xi_{ \pm}^{\prime}(x)>0$ and

It follows that

$$
\xi_{ \pm}^{\prime}(x)^{2} \xi_{ \pm}(x)=v(x)-v\left(x_{0}\right)
$$

$$
\xi_{ \pm}^{\prime}\left(x_{0}\right)= \pm\left|v^{\prime}\left(x_{0}\right)\right|^{1 / 3}
$$

Now we are in a position to construct the solutions $u_{ \pm}(x ; \hbar)$ of equation (1.1). The following assertion is well known (see, e.g., Theorem 2.5 in [25]).

Theorem 2.2. Let Assumption 2.1 hold for the sign " \pm ". Then the equation (1.1) where $\lambda=v\left(x_{0}\right)$ has the solution $u_{ \pm}(x ; \hbar)=u_{ \pm}\left(x ; x_{0}, \hbar\right)$ such that

(i) If $x \in\left[x_{0}, x_{2}\right)$ for $u_{+}$and $x \in\left(x_{1}, x_{0}\right]$ for $u_{-}$, then

$$
u_{ \pm}(x ; \hbar)=\pi^{1 / 2} \hbar^{-1 / 6}\left|\xi_{ \pm}^{\prime}(x)\right|^{-1 / 2} \mathrm{Ai}\left(\hbar^{-2 / 3} \xi_{ \pm}(x)\right)(1+O(\hbar))
$$

(ii) If $x \in\left(x_{1}, x_{0}\right]$ for $u_{+}$and $x \in\left[x_{0}, x_{2}\right)$ for $u_{-}$, then

$$
u_{ \pm}(x ; \hbar)=\pi^{1 / 2} \hbar^{-1 / 6}\left|\xi_{ \pm}^{\prime}(x)\right|^{-1 / 2} \operatorname{Ai}\left(\hbar^{-2 / 3} \xi_{ \pm}(x)\right)+O\left(\hbar\left(1+\hbar^{-2 / 3}\left|x-x_{0}\right|\right)^{-1 / 4}\right) .
$$

These estimates are uniform on compact subintervals of $\left(x_{1}, x_{2}\right)$.

We emphasize that formulas (2.9) and (2.10) differ by the estimates of the remainders only. Away from the point $x_{0}$, we can replace the Airy function Ai $(t)$ by its asymptotics (2.2) or (2.3). Indeed, in view of (2.8), we have

$$
\left|\xi_{ \pm}(x)\right| \geq c\left|x-x_{0}\right|, \quad c>0
$$

and hence $\hbar^{-2 / 3} \xi_{+}(x) \rightarrow \pm \infty$ if $\hbar^{-2 / 3}\left(x-x_{0}\right) \rightarrow \pm \infty$ and $\hbar^{-2 / 3} \xi_{-}(x) \rightarrow \pm \infty$ if $\hbar^{-2 / 3}\left(x-x_{0}\right) \rightarrow$ $\mp \infty$. This leads to the following result.

Corollary 2.3. The functions $u_{ \pm}$have the following asymptotic behavior as $\hbar \rightarrow 0$ :

(i) If $x>x_{0}$ for $u_{+}$and $x<x_{0}$ for $u_{-}$, then

$$
u_{ \pm}(x ; \hbar)=2^{-1}\left(v(x)-v\left(x_{0}\right)\right)^{-1 / 4} \exp \left(\mp \hbar^{-1} \int_{x_{0}}^{x}\left(v(y)-v\left(x_{0}\right)\right)^{1 / 2} d y\right)(1+O(\hbar))
$$

(ii) If $x<x_{0}$ for $u_{+}$and $x>x_{0}$ for $u_{-}$, then

$$
u_{ \pm}(x ; \hbar)=\left(v\left(x_{0}\right)-v(x)\right)^{-1 / 4} \sin \left( \pm \hbar^{-1} \int_{x}^{x_{0}}\left(v\left(x_{0}\right)-v(y)\right)^{1 / 2} d y+\pi / 4\right)+O(\hbar) .
$$

These estimates are uniform in $x$ on compact subintervals of $\left(x_{1}, x_{0}\right)$ and $\left(x_{0}, x_{2}\right)$.

Remark 2.4. Formulas (2.12) and (2.13) permit that $x$ approaches to $x_{0}$ as $\hbar \rightarrow 0$ but not too rapidly. To be precise, it suffices to require that $x$ lies away of a $O\left(\hbar^{2 / 3}\right)$ neighborhood of the point $x_{0}$. In this case the remainders in (2.12) and (2.13) should be replaced by $O\left(\hbar\left|x-x_{0}\right|^{-3 / 2}\right)$ and $O\left(\hbar\left|x-x_{0}\right|^{-7 / 4}\right)$, respectively.

On the other hand, using estimates (2.11) and $|\mathrm{Ai}(t)| \leq C(1+|t|)^{-1 / 4}$, we obtain uniform in $\hbar$ estimates of the functions $u_{ \pm}(x ; \hbar)$ in neighborhoods of the turning points.

Corollary 2.5. For all $x \in\left(x_{1}, x_{2}\right)$, the estimate

$$
\left|u_{ \pm}(x ; \hbar)\right| \leq C\left(\hbar^{2 / 3}+\left|x-x_{0}\right|\right)^{-1 / 4}
$$

holds with a constant $C$ that does not depend on $\hbar$. 
Note that all asymptotic relations (2.9), (2.10), (2.12) and (2.13) can be differentiated in $x$. In particular, we have the asymptotics

$$
u_{ \pm}^{\prime}(x ; \hbar)=\mp \pi^{-1 / 2} \hbar^{-1}\left(v\left(x_{0}\right)-v(x)\right)^{1 / 4} \cos \left( \pm \hbar^{-1} \int_{x}^{x_{0}}\left(v\left(x_{0}\right)-v(y)\right)^{1 / 2} d y+\pi / 4\right)+O(1)
$$

as $\hbar \rightarrow 0$ for $u_{+}$if $x<x_{0}$ and for $u_{-}$if $x>x_{0}$.

All these relations can also be differentiated with respect to $x_{0}$. For example, we have (recall that $\left.\lambda=v\left(x_{0}\right)\right)$

$$
\begin{aligned}
\partial u_{ \pm}\left(x ; x_{0}, \hbar\right) / \partial x_{0}= \pm 2^{-1} \pi^{-1 / 2} & \hbar^{-1}\left(v\left(x_{0}\right)-v(x)\right)^{-1 / 4} \int_{x}^{x_{0}}\left(v\left(x_{0}\right)-v(y)\right)^{-1 / 2} d y \\
& \times v^{\prime}\left(x_{0}\right) \cos \left( \pm \hbar^{-1} \int_{x}^{x_{0}}\left(v\left(x_{0}\right)-v(y)\right)^{1 / 2} d y+\pi / 4\right)+O(1)
\end{aligned}
$$

as $\hbar \rightarrow 0$ for $u_{+}$if $x<x_{0}$ and for $u_{-}$if $x>x_{0}$.

We also have to look at the behavior of the solutions $u_{ \pm}(x ; \hbar)$ as $x \rightarrow \pm \infty$. To that end, we need some weak condition on the behavior of the function $v(x)$ at infinity. The following result is a particular case of Theorem 2.5 in [25].

Theorem 2.6. Let the assumptions of Theorem 2.2 hold. Suppose that condition (1.10) is satisfied with $\lambda_{0}=v\left(x_{0}\right)$ and that, for some $\rho_{0}>1$, the function

$$
\left(\left|v(x)-\lambda_{0}\right|^{-3} v^{\prime}(x)^{2}+\left|v(x)-\lambda_{0}\right|^{-2}\left|v^{\prime \prime}(x)\right|\right)\left|\int_{x_{0}}^{x}\right| v(y)-\left.\left.\lambda_{0}\right|^{1 / 2} d y\right|^{\rho_{0}}
$$

is bounded as $x \rightarrow \pm \infty$. Then

$$
u_{ \pm}(\hbar) \in L^{2}\left(\mathbb{R}_{ \pm}\right) .
$$

Function (2.14) is bounded in all reasonable cases. For example, if $v(x) \rightarrow v_{0}>v\left(x_{0}\right)$, it is sufficient to require that

$$
v^{\prime}(x)^{2}+\left|v^{\prime \prime}(x)\right|=O\left(|x|^{-\rho_{0}}\right), \quad \rho_{0}>1, \quad|x| \rightarrow \infty .
$$

It is also satisfied if $v(x)$ behaves at infinity as $|x|^{\alpha}$ or $e^{\alpha|x|}$ where $\alpha>0$; in these cases $\rho_{0}=2$.

2.3. Next we consider the solutions of the equation (1.1) that are exponentially growing as $\hbar \rightarrow 0$ inside the barrier. The following assertion complements Theorem 2.2. Its proof will be given in the next section.

Theorem 2.7. Let Assumption 2.1 hold for the sign " \pm ". Then the equation (1.1) where $\lambda=v\left(x_{0}\right)$ has the solution $w_{ \pm}(x ; \hbar)=w_{ \pm}\left(x ; x_{0}, \hbar\right)$ such that

(i) If $x \in\left[x_{0}, x_{2}\right)$ for $w_{+}$and $x \in\left(x_{1}, x_{0}\right]$ for $w_{-}$, then

$$
w_{ \pm}(x ; \hbar)=\pi^{1 / 2} \hbar^{-1 / 6}\left|\xi_{ \pm}^{\prime}(x)\right|^{-1 / 2} \operatorname{Bi}\left(\hbar^{-2 / 3} \xi_{ \pm}(x)\right)(1+O(\hbar)) .
$$

(ii) If $x \in\left(x_{1}, x_{0}\right]$ for $w_{+}$and $x \in\left[x_{0}, x_{2}\right)$ for $w_{-}$, then

$$
w_{ \pm}(x ; \hbar)=\pi^{1 / 2} \hbar^{-1 / 6}\left|\xi_{ \pm}^{\prime}(x)\right|^{-1 / 2} \operatorname{Bi}\left(\hbar^{-2 / 3} \xi_{ \pm}(x)\right)+O\left(\hbar\left(1+\hbar^{-2 / 3}\left|x-x_{0}\right|\right)^{-1 / 4}\right) .
$$

These estimates are uniform on compact subintervals of $\left(x_{1}, x_{2}\right)$.

Obviously, formulas (2.15) and (2.16) differ by the remainders only. Using estimate (2.11) away from the points $x_{0}$, we can replace the Airy function $\mathrm{Bi}(t)$ by its asymptotics (2.4) or (2.5). This leads to the following result (cf. Corollary 2.3).

Corollary 2.8. The functions $w_{ \pm}$have the following asymptotic behavior as $\hbar \rightarrow 0$ : 
(i) If $x>x_{0}$ for $w_{+}$and $x<x_{0}$ for $w_{-}$, then

$$
w_{ \pm}(x ; \hbar)=\left(v(x)-v\left(x_{0}\right)\right)^{-1 / 4} \exp \left( \pm \hbar^{-1} \int_{x_{0}}^{x}\left(v(y)-v\left(x_{0}\right)\right)^{1 / 2} d y\right)(1+O(\hbar)) .
$$

(ii) If $x<x_{0}$ for $w_{+}$and $x>x_{0}$ for $w_{-}$, then

$$
w_{ \pm}(x ; \hbar)=\left(v\left(x_{0}\right)-v(x)\right)^{-1 / 4} \cos \left( \pm \hbar^{-1} \int_{x}^{x_{0}}\left(v\left(x_{0}\right)-v(y)\right)^{1 / 2} d y+\pi / 4\right)+O(\hbar) .
$$

These estimates are uniform in $x$ on compact subintervals of $\left(x_{1}, x_{0}\right)$ and $\left(x_{0}, x_{2}\right)$.

Remark 2.9. (i) Similarly to Theorem 2.2, the estimate of the remainders in (2.16) can be improved.

(ii) Formulas (2.17) and (2.18) permit that $x$ approaches to $x_{0}$ as $\hbar \rightarrow 0$ but not too rapidly. To be precise, it suffices to require that $x$ lies away of a $O\left(\hbar^{2 / 3}\right)$ neighborhood of the point $x_{0}$. In this case the remainders in (2.17) and (2.18) should be replaced by $O\left(\hbar\left|x-x_{0}\right|^{-3 / 2}\right)$ and $O\left(\hbar^{7 / 6}\left|x-x_{0}\right|^{-7 / 4}\right)$, respectively.

(iii) Corollary 2.5 remains true for the functions $w_{ \pm}$.

(iv) All asymptotic formulas (2.15), (2.16) and (2.17), (2.18) may be differentiated in $x$ (and in $\left.x_{0}\right)$. For derivatives, the remainders $O(\hbar)$ should be replaced by $O(1)$.

Of course the solutions $u_{+}, w_{+}$and $u_{-}, w_{-}$of equation (1.1) are linearly independent. Both pairs of formulas (2.12), (2.17) or (2.13), (2.18) allow one to calculate their Wronskians:

$$
\left\{u_{ \pm}(\hbar), w_{ \pm}(\hbar)\right\}:=u_{ \pm}^{\prime}(x, \hbar) w_{ \pm}(x, \hbar)-u_{ \pm}(x, \hbar) w_{ \pm}^{\prime}(x, \hbar)=\mp \hbar^{-1}(1+O(\hbar)) .
$$

2.4. An arbitrary real solution $\psi$ of equation (1.1) on the interval $\left(x_{1}, x_{2}\right)$ is a linear combination of the solutions $u_{+}, w_{+}$and $u_{-}, w_{-}$, that is,

$$
\psi(x, \hbar)=\alpha_{ \pm}(\hbar) u_{ \pm}(x, \hbar)+\beta_{ \pm}(\hbar) w_{ \pm}(x, \hbar) .
$$

It is convenient to put $A_{ \pm}(\hbar)=\sqrt{\alpha_{ \pm}(\hbar)^{2}+\beta_{ \pm}(\hbar)^{2}}$ and

$$
\alpha_{ \pm}(\hbar)=A_{ \pm}(\hbar) \cos \vartheta_{ \pm}(\hbar), \quad \beta_{ \pm}(\hbar)=A_{ \pm}(\hbar) \sin \vartheta_{ \pm}(\hbar) ;
$$

the phases $\vartheta_{ \pm}(\hbar)$ are of course defined up to terms $2 \pi n$ where $n \in \mathbb{Z}$. If Assumption 2.1 holds for the sign " + ", then it follows from formulas (2.13), (2.18) that $\psi$ has the asymptotic behavior

$$
\psi(x ; \hbar)=A_{+}(\hbar)(\lambda-v(x))^{-1 / 4}\left(\sin \left(\hbar^{-1} \int_{x}^{x_{0}}(\lambda-v(y))^{1 / 2} d y+\frac{\pi}{4}+\vartheta_{+}(\hbar)\right)+O(\hbar)\right)
$$

for $x \in\left(x_{1}, x_{0}\right)$. Similarly, if Assumption 2.1 holds for the sign " - ", then $\psi$ has the asymptotic behavior

$$
\psi(x ; \hbar)=A_{-}(\hbar)(\lambda-v(x))^{-1 / 4}\left(\sin \left(\hbar^{-1} \int_{x_{0}}^{x}(\lambda-v(y))^{1 / 2} d y+\frac{\pi}{4}+\vartheta_{-}(\hbar)\right)+O(\hbar)\right)
$$

for $x \in\left(x_{0}, x_{2}\right)$. Of course relations (2.20) and (2.21) are uniform in $x$ on compact subintervals of $\left(x_{1}, x_{0}\right)$ and $\left(x_{0}, x_{2}\right)$, respectively.

\section{Proof of Theorem 2.7}

3.1. We will prove Theorem 2.7 for the sign " + " and omit this index. Let the function $\xi(x)=\xi_{+}\left(x ; x_{0}\right)$ be defined by formulas (2.6), and let $\xi_{j}=\xi\left(x_{j}\right)$ for $j=1,2$. Since $\xi^{\prime}(x)>0$, the variable $x$ can be considered as a function of $\xi$ for $\xi \in\left(\xi_{1}, \xi_{2}\right)$.

Let us make the change of variables $x \mapsto \xi$ in equation (1.1) for $w(x ; \hbar)$ and set

$$
w(x ; \hbar)=\pi^{1 / 2} \hbar^{-1 / 6} \xi^{\prime}(x)^{-1 / 2} f\left(\hbar^{-2 / 3} \xi(x) ; \hbar\right) .
$$


Then using identity (2.7), we obtain that

$$
-f^{\prime \prime}\left(\hbar^{-2 / 3} \xi ; \hbar\right)+\hbar^{-2 / 3} \xi f\left(\hbar^{-2 / 3} \xi ; \hbar\right)=\hbar^{4 / 3} r(\xi) f\left(\hbar^{-2 / 3} \xi ; \hbar\right),
$$

where

$$
r(\xi)=p(x(\xi))
$$

and

$$
p(x)=\left(\xi^{\prime}(x)^{-1 / 2}\right)^{\prime \prime} \xi^{\prime}(x)^{-3 / 2}
$$

is a continuous function of $x \in\left(x_{1}, x_{2}\right)$. Setting in (3.2) $t=\hbar^{-2 / 3} \xi$, we get the following intermediary result.

Lemma 3.1. Let $t=\hbar^{-2 / 3} \xi(x)$, and let the functions $w(x ; \hbar)$ and $f(t ; \hbar)$ be related by formula (3.1). Then equation (1.1) for $w(x ; \hbar)$ and $x \in\left(x_{1}, x_{2}\right)$ is equivalent to the equation

$$
-f^{\prime \prime}(t ; \hbar)+t f(t ; \hbar)=R(t ; \hbar) f(t ; \hbar)
$$

for $f(t ; \hbar)$ and $t \in\left(\xi_{1} \hbar^{-2 / 3}, \xi_{2} \hbar^{-2 / 3}\right)$. Here

$$
R(t ; \hbar)=\hbar^{4 / 3} r\left(\hbar^{2 / 3} t\right)
$$

and the function $r(\xi)$ is defined by formulas (3.3) and (3.4).

Since $r(\xi)$ is a bounded function, we have the estimate

$$
|R(t ; \hbar)| \leq C \hbar^{4 / 3}
$$

where for all $\eta_{1}>\xi_{1}$ and $\eta_{2}<\xi_{2}$, the constant $C=C\left(\eta_{1}, \eta_{2}\right)$ does not depend on $t \in$ $\left(\eta_{1} \hbar^{-2 / 3}, \eta_{2} \hbar^{-2 / 3}\right)$.

3.2. Let us reduce differential equation (3.5) to a Volterra integral equation. Set

$$
K(t, s ; \hbar)=\pi(\operatorname{Ai}(t) \operatorname{Bi}(s)-\operatorname{Ai}(s) \operatorname{Bi}(t)) R(s ; \hbar)
$$

and consider the equation

$$
f(t ; \hbar)=\operatorname{Bi}(t)+\int_{0}^{t} K(t, s ; \hbar) f(s ; \hbar) d s .
$$

Differentiating it twice, we see that its solution satisfies also differential equation (3.5). We will study equation (3.7) separately for $t \leq 0$ and $t \geq 0$.

If $t \leq 0$, we use that

$$
|\mathrm{Ai}(t)|+|\operatorname{Bi}(t)| \leq C(1+|t|)^{-1 / 4}
$$

according to (2.2) and (2.5). Hence, by virtue of (3.6), we have the estimate

$$
|K(t, s ; \hbar)| \leq C \hbar^{4 / 3}(1+|s|)^{-1 / 2}, \quad t \leq s \leq 0 .
$$

We consider equation (3.7) on the interval $\left(\eta_{1} \hbar^{-2 / 3}, 0\right)$ in the space $\mathbf{B}(\hbar)$ of functions $f$ with the norm

$$
\|f\|=\sup _{t \in\left(\eta_{1} \hbar^{-2 / 3}, 0\right)}(1+|t|)^{1 / 4}|f(t)| .
$$

Let us introduce an auxiliary operator $\mathbf{K}(\hbar)$ in this space by the formula

$$
(\mathbf{K}(\hbar) f)(t)=\int_{0}^{t} K(t, s ; \hbar) f(s) d s
$$

In view of (3.8) we have

$$
|(\mathbf{K}(\hbar) f)(t)| \leq C \hbar^{4 / 3}(1+|t|)^{1 / 4}\|f\| \leq C_{1} \hbar(1+|t|)^{-1 / 4}\|f\|
$$


if $t \in\left(\eta_{1} \hbar^{-2 / 3}, 0\right)$ so that $\|\mathbf{K}(\hbar)\|=O(\hbar)$. It follows that equation (3.7) has a unique solution $f(t ; \hbar)$ and

$$
|f(t ; \hbar)-\operatorname{Bi}(t)| \leq C \hbar(1+|t|)^{-1 / 4} \quad \text { if } \quad t \in\left(\eta_{1} \hbar^{-2 / 3}, 0\right) .
$$

According to (2.11), this yields representation (2.16) for function (3.1).

For $t \geq 0$, we make the multiplicative change of variables

$$
f(t ; \hbar)=\pi^{1 / 2} \hbar^{-1 / 6} \operatorname{Bi}(t) g(t ; \hbar) .
$$

Then equation (3.7) reads as

$$
g(t ; \hbar)=1+\int_{0}^{t} L(t, s ; \hbar) g(s ; \hbar) d s
$$

where

$$
L(t, s ; \hbar)=\pi\left(\frac{\operatorname{Ai}(t)}{\operatorname{Bi}(t)} \operatorname{Bi}(s)^{2}-\operatorname{Ai}(s) \operatorname{Bi}(s)\right) R(s ; \hbar), \quad 0 \leq s \leq t .
$$

It follows from relations (2.2) and (2.5) that

$$
|\operatorname{Ai}(s) \operatorname{Bi}(s)| \leq C(1+s)^{-1 / 2} .
$$

Since $\mathrm{Bi}(t)$ is an increasing function of $t>0$, this yields the estimate (cf. (3.8))

$$
|L(t, s ; \hbar)| \leq C \hbar^{4 / 3}(1+s)^{-1 / 2}, \quad 0 \leq s \leq t .
$$

So quite similarly to the case $t \leq 0$, we get the bound (cf (3.9) )

$$
|g(t ; \hbar)-1| \leq C \hbar^{4 / 3}(1+t)^{1 / 2} \leq C_{1} \hbar \quad \text { if } \quad t \in\left(0, \eta_{2} \hbar^{-2 / 3}\right) .
$$

Using (3.10), we see that function (3.1) satisfies estimate (2.15). Differentiating integral equation (3.7) and (3.11) with respect to $t$, we obtain asymptotic relations for $g^{\prime}(t ; \hbar)$ and then for $w^{\prime}(x ; \hbar)$. This concludes the proof of Theorem 2.7.

\section{One Potential Well}

4.1. Here we consider one potential well $\left(x_{-}, x_{+}\right)$bounded by the turning points $x_{-}=x_{-}(\lambda)$ and $x_{+}=x_{+}(\lambda)$. To be precise, we accept

Assumption 4.1. The function $v \in C^{2}$ on some interval $\left(x_{1}, x_{2}\right) \subset \mathbb{R}$. The equation $v(x)=\lambda_{0}$ has two solutions $x_{+}=x_{+}\left(\lambda_{0}\right), x_{-}=x_{-}\left(\lambda_{0}\right)$ in $\left(x_{1}, x_{2}\right), x_{-}<x_{+}$and $v(x)<\lambda_{0}$ for $x \in\left(x_{-}, x_{+}\right)$, $v(x)>\lambda_{0}$ for $x \notin\left[x_{-}, x_{+}\right]$. Moreover, the function $v$ belongs to the class $C^{3}$ in some neighborhood of the points $x_{-}, x_{+}$and $v^{\prime}\left(x_{-}\right)<0, v^{\prime}\left(x_{+}\right)>0$.

Set

$$
\Phi(\lambda)=\int_{x_{-}(\lambda)}^{x_{+}(\lambda)}(\lambda-v(x))^{1 / 2} d x
$$

It is easy to see that $\Phi \in C^{1}\left(\Lambda_{1}, \Lambda_{2}\right)$. Moreover, differentiating formula (4.1) and using the equation $\lambda-v\left(x_{ \pm}(\lambda)\right)=0$, we obtain that

$$
\dot{\Phi}(\lambda)=2^{-1} \int_{x_{-}(\lambda)}^{x_{+}(\lambda)}(\lambda-v(x))^{-1 / 2} d x>0 .
$$

Thus, $\Phi$ is a one-to-one mapping of a neighborhood $\left(\Lambda_{1}, \Lambda_{2}\right)$ of $\lambda_{0}$ onto a neighborhood $\left(\Phi\left(\Lambda_{1}\right), \Phi\left(\Lambda_{2}\right)\right)$ of the point $\mu_{0}=\Phi\left(\lambda_{0}\right)$.

Note that a classical particle of the energy $\lambda$ and the mass $m=1 / 2$ oscillates between the points $x_{-}(\lambda)$ and $x_{+}(\lambda)$ (see the book [16, $\S 11$ and $\S 44$ ). The function $\Phi(\lambda)$ is known as the abbreviated action (with the coefficient $1 / 2$ ) of this motion, and the function $\dot{\Phi}(\lambda)$ is the half-period of the oscillations. 
We are interested in the asymptotic behavior as $\hbar \rightarrow 0$ of an arbitrary real solution $\psi(x ; \hbar)$ of equation (1.1) on the interval $\left(x_{-}, x_{+}\right)$. Recall that the functions $u_{ \pm}\left(x ; x_{ \pm}, \hbar\right)$ and $w_{ \pm}\left(x ; x_{ \pm}, \hbar\right)$ are constructed in Theorems 2.2 and 2.7, respectively. Since according to (2.19) the functions $u_{+}\left(x ; x_{+}, \hbar\right), w_{+}\left(x ; x_{+}, \hbar\right)$ as well as $u_{-}\left(x ; x_{-}, \hbar\right), w_{-}\left(x ; x_{-}, \hbar\right)$ are linearly independent, we have

$$
\begin{aligned}
\psi(x, \hbar) & =\alpha_{+}(\hbar) u_{+}\left(x ; x_{+}, \hbar\right)+\beta_{+}(\hbar) w_{+}\left(x ; x_{+}, \hbar\right) \\
& =\alpha_{-}(\hbar) u_{-}\left(x ; x_{-}, \hbar\right)+\beta_{-}(\hbar) w_{-}\left(x ; x_{-}, \hbar\right)
\end{aligned}
$$

for some $\alpha_{ \pm}(\hbar), \beta_{ \pm}(\hbar) \in \mathbb{R}$. We put $A_{ \pm}(\hbar)=\sqrt{\alpha_{ \pm}(\hbar)^{2}+\beta_{ \pm}(\hbar)^{2}}$ and

$$
\alpha_{ \pm}(\hbar)=A_{ \pm}(\hbar) \cos \phi_{ \pm}(\hbar), \quad \beta_{ \pm}(\hbar)=A_{ \pm}(\hbar) \sin \phi_{ \pm}(\hbar), \quad \phi_{ \pm}(\hbar) \in \mathbb{R} /(2 \pi \mathbb{Z}) .
$$

Let the functions $\Phi_{ \pm}(x ; \lambda)$ be defined by formula (1.2), and let $\lambda=v\left(x_{ \pm}\right)$. It follows from relations (2.13) and (2.18) where $x_{0}=x_{+}$and $x_{0}=x_{-}$that the asymptotic behavior of $\psi(x ; \hbar)$ for $x \in\left(x_{-}, x_{+}\right)$is given by formulas (1.3) and (1.4) where

$$
\varphi_{ \pm}(\hbar)=\phi_{ \pm}(\hbar)+\pi / 4 \text {. }
$$

Of course formulas (1.3) and (1.4) are uniform in $x$ on compact subintervals of $\left(x_{-}, x_{+}\right)$and can be differentiated in $x$; in the asymptotic formulas for the derivatives, the remainders $O(\hbar)$ should be replaced by $O(1)$.

The phases $\varphi_{+}(\hbar), \varphi_{-}(\hbar)$ in formulas (1.3) and (1.4) are linked by some relations that we will now derive. The arguments below are essentially the same as those used for the derivation of the Bohr-Sommerfeld quantization condition (see, e.g., Subsection 4.1 of [25]).

First, we calculate various Wronskians. The next assertion follows from asymptotic formulas (2.13) and (2.18) for $u_{ \pm}\left(x ; x_{ \pm}(\lambda), \hbar\right)$ and $w_{ \pm}\left(x ; x_{ \pm}(\lambda), \hbar\right)$. These formulas can be differentiated both in $x$ and $\lambda$. We also use that

$$
\Phi(\lambda)=\Phi_{+}(x ; \lambda)+\Phi_{-}(x ; \lambda) .
$$

Lemma 4.2. Let Assumption 4.1 hold, and let the function $\Phi$ be defined by formula (4.1). Then, as $\hbar \rightarrow 0$,

$$
\begin{gathered}
\left\{u_{-}\left(x_{-}, \hbar\right), u_{+}\left(x_{+}, \hbar\right)\right\}=\hbar^{-1} \cos \left(\hbar^{-1} \Phi\right)+O(1), \\
\left\{u_{-}\left(x_{-}, \hbar\right), w_{+}\left(x_{+}, \hbar\right)\right\}=-\hbar^{-1} \sin \left(\hbar^{-1} \Phi\right)+O(1), \\
\left\{w_{-}\left(x_{-}, \hbar\right), u_{+}\left(x_{+}, \hbar\right)\right\}=-\hbar^{-1} \sin \left(\hbar^{-1} \Phi\right)+O(1),
\end{gathered}
$$

and

$$
\left\{w_{-}\left(x_{-}, \hbar\right), w_{+}\left(x_{+}, \hbar\right)\right\}=-\hbar^{-1} \cos \left(\hbar^{-1} \Phi\right)+O(1)
$$

All these formulas can be differentiated in $\lambda$; in this case the remainders $O(1)$ should be replaced by $O\left(\hbar^{-1}\right)$.

For short, we set $u_{ \pm}(\hbar)=u_{ \pm}\left(x_{ \pm}, \hbar\right), w_{ \pm}(\hbar)=w_{ \pm}\left(x_{ \pm}, \hbar\right)$. In view of (4.4) we have

$$
\begin{aligned}
\left(A_{+}(\hbar) A_{-}(\hbar)\right)^{-1}\left\{\alpha_{+}(\hbar) u_{+}(\hbar)+\beta_{+}(\hbar) w_{+}(\hbar), \alpha_{-}(\hbar) u_{-}(\hbar)+\beta_{-}(\hbar) w_{-}(\hbar)\right\} & \\
= & \cos \phi_{+}(\hbar) \cos \phi_{-}(\hbar)\left\{u_{+}(\hbar), u_{-}(\hbar)\right\}+\sin \phi_{+}(\hbar) \sin \phi_{-}(\hbar)\left\{w_{+}(\hbar), w_{-}(\hbar)\right\} \\
& +\cos \phi_{+}(\hbar) \sin \phi_{-}(\hbar)\left\{u_{+}(\hbar), w_{-}(\hbar)\right\}+\sin \phi_{+}(\hbar) \cos \phi_{-}(\hbar)\left\{w_{+}(\hbar), u_{-}(\hbar)\right\} .
\end{aligned}
$$

Put

$$
\varphi(\lambda, \hbar)=\varphi_{+}(\lambda, \hbar)+\varphi_{-}(\lambda, \hbar)=\phi_{+}(\lambda, \hbar)+\phi_{-}(\lambda, \hbar)+\pi / 2 .
$$


According to Lemma 4.2 expression (4.7) equals

$$
\begin{gathered}
\hbar^{-1}\left(-\cos \phi_{+}(\hbar) \cos \phi_{-}(\hbar)+\sin \phi_{+}(\hbar) \sin \phi_{-}(\hbar)\right) \cos \left(\hbar^{-1} \Phi\right) \\
+\hbar^{-1}\left(\cos \phi_{+}(\hbar) \sin \phi_{-}(\hbar)+\sin \phi_{+}(\hbar) \cos \phi_{-}(\hbar)\right) \sin \left(\hbar^{-1} \Phi\right)+O(1) \\
=\hbar^{-1}\left(-\sin \varphi(\hbar) \cos \left(\hbar^{-1} \Phi\right)-\cos \varphi(\hbar) \sin \left(\hbar^{-1} \Phi\right)\right)+O(1) \\
=-\hbar^{-1} \sin \left(\hbar^{-1} \Phi+\varphi(\hbar)\right)+O(1) .
\end{gathered}
$$

On the other hand, it follows from equality (4.3) that expression (4.7) is zero. This yields the equation

whence

$$
\sin \left(\hbar^{-1} \Phi(\lambda)+\varphi(\lambda, \hbar)\right)=O(\hbar)
$$

with some integer number $n=n(\lambda, \hbar)$.

$$
\Phi(\lambda)=(\pi n-\varphi(\lambda, \hbar)) \hbar+O\left(\hbar^{2}\right)
$$

Putting together relations (4.5), (4.8) and the quantization condition (4.10), we see that formula (1.3) can be rewritten as

$$
\psi_{+}(x ; \lambda, \hbar)=(-1)^{n} A_{+}(\hbar)(\lambda-v(x))^{-1 / 4}\left(\sin \left(\hbar^{-1} \Phi_{-}(x ; \lambda)+\varphi_{-}(\hbar)\right)+O(\hbar)\right) .
$$

Comparing it with formula (1.4), we find that

$$
A_{-}(\lambda, \hbar)=A_{+}(\lambda, \hbar)(1+O(\hbar))
$$

and that the integer $n$ in (4.10) is even.

Let us summarize the results obtained.

Theorem 4.3. Let Assumption 4.1 hold. Then:

(i) An arbitrary real solution $\psi(x ; \lambda, h)$ of equation (1.1) has both asymptotics (1.3) and (1.4) where the functions $\Phi_{ \pm}(x ; \lambda)$ are defined by formula (1.2).

(ii) Functions (4.1) and (4.8) satisfy the conditions (4.9) and (4.10) with some integer even number $n=n(\lambda, \hbar)$.

(iii) The amplitudes $A_{+}(\lambda, \hbar), A_{-}(\lambda, \hbar)$ in (1.3), (1.4) are linked by relation (4.11).

4.2. If $\varphi(\lambda, \hbar)=\pi / 2(\bmod \pi)$, then formula (4.10) $)$ reduces to the Bohr-Sommerfeld quantization condition. In particular, this is true if the fixing conditions $\varphi_{ \pm}(\lambda, \hbar)=\pi / 4(\bmod \pi)$ are satisfied at both points $x_{ \pm}(\lambda)$. Let us state this result explicitly.

Theorem 4.4. Under Assumption 4.1 suppose that a real solution $\psi(x ; \lambda, h)$ of equation (1.1) has asymptotics (1.3) and (1.4) where both phases $\varphi_{ \pm}(\lambda, \hbar)=\pi / 4(\bmod \pi)$. Then function (4.1) satisfies the condition

$$
\cos \left(\hbar^{-1} \Phi(\lambda)\right)=O(\hbar)
$$

and hence

$$
\Phi(\lambda)=\pi(n+1 / 2) \hbar+O\left(\hbar^{2}\right)
$$

for some integer number $n=n(\lambda, \hbar)$.

Remark 4.5. It is possible that $\varphi_{ \pm}(\lambda, \hbar)=\pi / 4(\bmod \pi)$ only for $\hbar$ in some set $\Gamma \subset \mathbb{R}_{+}$such that $0 \in \operatorname{clos} \Gamma$. Then conditions (4.12) and (4.13) are also true for $h \in \Gamma$.

Of course in Theorem 4.4, $n$ may be an arbitrary integer. Indeed, if $\pi^{-1}\left(\varphi_{+}-\varphi_{-}\right)$is even (resp., odd), then Theorem 4.3(ii) implies (4.13) with $n$ even (resp., odd).

Theorem 4.4 can be supplemented by the following assertion. To state it, we need an additional assumption on the remainder $O\left(\hbar^{2}\right)$ in (4.13) . 
Proposition 4.6. Let Assumption 4.1 hold, and let $\lambda$ and $\Phi^{-1}(\pi(n+1 / 2) \hbar)$ belong to a neighborhood of $\lambda_{0}$. Suppose that

$$
\epsilon(\lambda, \hbar)=O\left(\hbar^{2}\right), \quad \dot{\epsilon}(\lambda, \hbar)=o(1) .
$$

Then for every integer $n$, there may exist only one value of $\lambda=\lambda_{n}(\hbar)$ satisfying the condition

$$
\Phi(\lambda)=\pi(n+1 / 2) \hbar+\epsilon(\lambda, \hbar) .
$$

Proof. Supposing that there are two different $\lambda^{\prime}(\hbar), \lambda^{\prime \prime}(\hbar)$ satisfying (4.15), we find a point $\nu(\hbar) \in$ $\left(\lambda^{\prime}(\hbar), \lambda^{\prime \prime}(\hbar)\right)$ such that

$$
\dot{\Phi}(\nu(\hbar))=\dot{\epsilon}(\nu(\hbar), \hbar) .
$$

According to (4.2) we have $\dot{\Phi}(\nu(\hbar)) \geq c>0$, while according to (4.14) the right-hand side tends to 0 as $\hbar \rightarrow 0$. So, equality (4.16) leads to the contradiction.

The following result is converse to Theorem 4.4.

Proposition 4.7. Let Assumption 4.1 hold. Suppose that a number $\pi(n+1 / 2) \hbar$ belongs to a neighborhood of $\Phi\left(\lambda_{0}\right)$. Then there exists the value of $\tilde{\lambda}=\tilde{\lambda}_{n}(\hbar)$ (that is, it depends on $n$ and $\hbar$ ) of $\lambda$ such that relations (4.13) and

$$
u_{-}\left(x ; x_{-}(\tilde{\lambda}), \hbar\right)=a(\tilde{\lambda}, \hbar) u_{+}\left(x ; x_{+}(\tilde{\lambda}), \hbar\right)
$$

hold true. Moreover, the coefficient a in (4.17) satisfies

$$
a(\tilde{\lambda}, \hbar)=(-1)^{n}+O(\hbar) .
$$

Proof. In view of formula (4.6) the Wronskian of the solutions $u_{+}\left(x ; x_{+}(\lambda), \hbar\right)$ and $u_{-}\left(x ; x_{-}(\lambda), \hbar\right)$ of the equation (1.1) is given by the formula

$$
\left\{u_{-}\left(x_{-}(\lambda), \hbar\right), u_{+}\left(x_{+}(\lambda), \hbar\right)\right\}=\hbar^{-1} \cos \left(\hbar^{-1} \Phi(\lambda)\right)+\varepsilon(\lambda, \hbar)
$$

where $\epsilon(\lambda, \hbar)$ is a continuous function of $\lambda \in\left(\Lambda_{1}, \Lambda_{2}\right)$ and $\varepsilon(\lambda, \hbar)=O(1)$ as $\hbar \rightarrow 0$. Expression (4.19) is zero for some $\tilde{\lambda}=\tilde{\lambda}_{n}(\hbar)$ obeying condition (4.13) whence (4.17) follows. According to Corollary 2.3(ii) the asymptotics of $u_{+}\left(x ; x_{+}(\tilde{\lambda}), \hbar\right)$ and $u_{-}\left(x ; x_{-}(\tilde{\lambda}), \hbar\right)$ as $\hbar \rightarrow 0$ are given by formulas (1.3), (1.4) where $A_{ \pm}=1$ and $\varphi_{ \pm}=\pi / 4$. Comparing them, we obtain relation (4.18).

\section{Passage through a potential Barrier. Fixing Conditions}

Here we show that, at least from one side, a potential barrier fixes the phase of a real solution of equation (1.1) in the same way as a barrier of infinite length.

5.1. Let us accept the following

Assumption 5.1. The function $v \in C^{2}$ on some interval $\left(x_{1}, x_{2}\right) \subset \mathbb{R}$. The equation $v(x)=\lambda_{0}$ has two solutions $b_{1}=b_{1}\left(\lambda_{0}\right), b_{2}=b_{2}\left(\lambda_{0}\right)$ in $\left(x_{1}, x_{2}\right), b_{1}<b_{2}$ and $v(x)>\lambda$ for $x \in\left(b_{1}, b_{2}\right)$, $v(x)<\lambda$ for $x \notin\left[b_{1}, b_{2}\right]$. Moreover, the function $v$ belongs to the class $C^{3}$ in some neighborhoods of the points $b_{1}, b_{2}$ and $v^{\prime}\left(b_{1}\right)>0, v^{\prime}\left(b_{2}\right)<0$. 


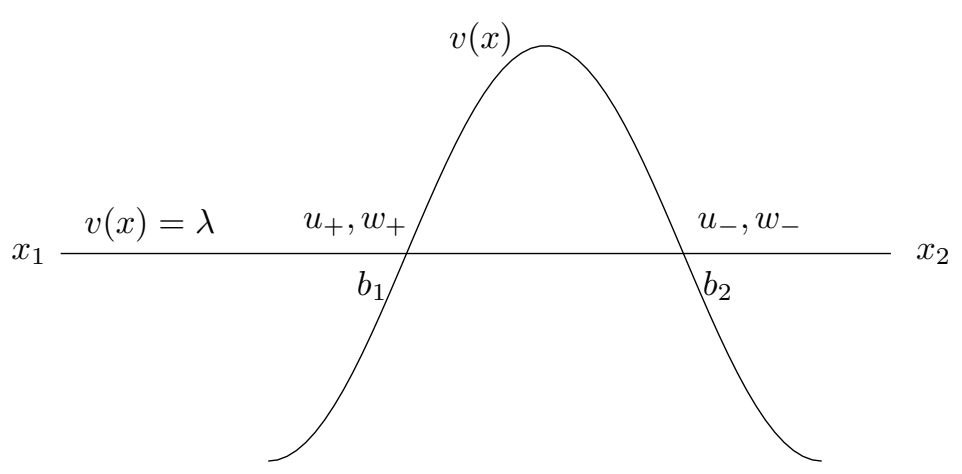

Potential barrier between $b_{1}$ and $b_{2}$

Thus we have a potential barrier between the points $b_{1}$ and $b_{2}$. The energy

$$
\lambda=v\left(b_{1}\right)=v\left(b_{2}\right) \in\left(\Lambda_{1}, \Lambda_{2}\right)
$$

is fixed. We are interested in the asymptotic behavior as $\hbar \rightarrow 0$ of an arbitrary real solution $\psi(x ; \hbar)$ of equation (1.1) on the interval $\left(x_{1}, x_{2}\right)$. Since according to (2.19) the functions $u_{+}\left(x ; b_{1}, \hbar\right)$, $w_{+}\left(x ; b_{1}, \hbar\right)$ and $u_{-}\left(x ; b_{2}, \hbar\right), w_{-}\left(x ; b_{2}, \hbar\right)$ (these functions are constructed in Theorems 2.2 and 2.7. respectively) are linearly independent, we have

$$
\begin{aligned}
\psi(x, \hbar) & =\alpha_{1}(\hbar) u_{+}\left(x ; b_{1}, \hbar\right)+\beta_{1}(\hbar) w_{+}\left(x ; b_{1}, \hbar\right) \\
& =\alpha_{2}(\hbar) u_{-}\left(x ; b_{2}, \hbar\right)+\beta_{2}(\hbar) w_{-}\left(x ; b_{2}, \hbar\right) .
\end{aligned}
$$

We put $A_{j}(\hbar)=\sqrt{\alpha_{j}(\hbar)^{2}+\beta_{j}(\hbar)^{2}}$ and

$$
\alpha_{j}(\hbar)=A_{j}(\hbar) \cos \vartheta_{j}(\hbar), \quad \beta_{j}(\hbar)=A_{j}(\hbar) \sin \vartheta_{j}(\hbar), \quad \theta_{j}(\hbar) \in \mathbb{R} /(2 \pi \mathbb{Z}), \quad j=1,2 .
$$

The phases $\vartheta_{1}(\hbar), \vartheta_{2}(\hbar)$ (and the amplitudes $\left.A_{1}(\hbar), A_{2}(\hbar)\right)$ in these formulas are linked by some relations that we will now derive. To that end, we need to calculate various Wronskians.

Lemma 5.2. Let Assumption 5.1 hold. Set

$$
\Omega(\lambda)=\int_{b_{1}(\lambda)}^{b_{2}(\lambda)} \sqrt{v(x)-\lambda} d x
$$

Then

$$
\begin{aligned}
\left\{u_{+}\left(b_{1}, \hbar\right), u_{-}\left(b_{2}, \hbar\right)\right\} & =-2^{-1} \hbar^{-1} e^{-\hbar^{-1} \Omega}(1+O(\hbar)), \\
\left\{w_{+}\left(b_{1}, \hbar\right), w_{-}\left(b_{2}, \hbar\right)\right\} & =2 \hbar^{-1} e^{\hbar^{-1} \Omega}(1+O(\hbar)) .
\end{aligned}
$$

and

$$
\left\{u_{ \pm}\left(b_{1}, \hbar\right), w_{\mp}\left(b_{2}, \hbar\right)\right\}=O\left(e^{-\hbar^{-1}(1-\varepsilon) \Omega}\right), \quad \forall \varepsilon>0 .
$$

Proof. We can calculate these Wronskians in an arbitrary point $x \in\left(b_{1}, b_{2}\right)$. Let us proceed from formulas (2.12) and (2.17) where $x_{0}=b_{1}$ or $x_{0}=b_{2}$ and $v\left(x_{0}\right)=\lambda$. These formulas can be differentiated in $x$. Relation (5.4) follows from (2.12), and relation (5.5) follows from (2.17). Using again (2.12) and (2.17), we find that

$$
\left\{u_{+}\left(b_{1}, \hbar\right), w_{-}\left(b_{2}, \hbar\right)\right\}=\hbar^{-1} O\left(\exp \left(-\hbar^{-1} \int_{b_{1}}^{x} \sqrt{v(x)-\lambda} d x+\hbar^{-1} \int_{x}^{b_{2}} \sqrt{v(x)-\lambda} d x\right)\right) .
$$

Since $x$ can be chosen arbitrary close to $b_{2}$, this proves (5.6) for the upper sign. The lower sign is considered quite similarly. 
5.2. It follows from (5.1) and (5.2) that

$$
\begin{aligned}
A_{1}(\hbar)\left(u_{+}\left(x ; b_{1}, \hbar\right) \cos \vartheta_{1}(\hbar)+w_{+}\right. & \left.\left(x ; b_{1}, \hbar\right) \sin \vartheta_{1}(\hbar)\right) \\
& =A_{2}(\hbar)\left(u_{-}\left(x ; b_{2}, \hbar\right) \cos \vartheta_{2}(\hbar)+w_{-}\left(x ; b_{2}, \hbar\right) \sin \vartheta_{2}(\hbar)\right) .
\end{aligned}
$$

Let us take the Wronskian of both sides of this equation with the function $u_{+}\left(x ; b_{1}, \hbar\right)$ :

$$
\begin{aligned}
A_{1}(\hbar)\left\{u_{+}\left(b_{1}, \hbar\right), w_{+}\left(b_{1}, \hbar\right)\right\} \sin \vartheta_{1}(\hbar) \\
=A_{2}(\hbar)\left(\left\{u_{+}\left(b_{1}, \hbar\right), u_{-}\left(b_{2}, \hbar\right)\right\} \cos \vartheta_{2}(\hbar)+\left\{u_{+}\left(b_{1}, \hbar\right), w_{-}\left(b_{2}, \hbar\right)\right\} \sin \vartheta_{2}(\hbar)\right) .
\end{aligned}
$$

Using relations (2.19), (5.4) and (5.6), we see that, for any $\varepsilon>0$,

$$
A_{1}(\hbar) \sin \vartheta_{1}(\hbar)=A_{2}(\hbar) O\left(e^{-\hbar^{-1}(1-\varepsilon) \Omega}\right) .
$$

Quite similarly, taking the Wronskian of equation (5.7) with the function $u_{-}\left(x ; b_{2}, \hbar\right)$, we find that

$$
A_{2}(\hbar) \sin \vartheta_{2}(\hbar)=A_{1}(\hbar) O\left(e^{-\hbar^{-1}(1-\varepsilon) \Omega}\right) .
$$

Multiplying equations (5.9) and (5.8) and neglecting the common factor $A_{1}(\hbar) A_{2}(\hbar)$, we obtain the following assertion.

Theorem 5.3. Let Assumption 5.1 hold. Then an arbitrary real solution of equation (1.1) is given by formulas (5.1) and (5.2) where the phases satisfy the estimate

$$
\sin \vartheta_{1}(\hbar) \sin \vartheta_{2}(\hbar)=O\left(e^{-2 \hbar^{-1}(1-\varepsilon) \Omega}\right), \quad \forall \varepsilon>0 .
$$

Corollary 5.4. For every $\hbar$, at least one of the phases satisfies the condition

$$
\sin \vartheta_{j}(\hbar)=O\left(e^{-\hbar^{-1}(1-\varepsilon) \Omega}\right), \quad j=1,2, \quad \forall \varepsilon>0 .
$$

Let us now come back to relations (5.1) and use asymptotics (2.13) and (2.18) for the functions $u_{ \pm}$and $w_{ \pm}$. Let the functions $\Theta_{j}(x, \lambda)$ be defined by formula (1.7), and put

$$
\theta_{j}(\hbar)=\vartheta_{j}(\hbar)+\pi / 4 \text {. }
$$

Since the phase shift $O\left(e^{-\hbar^{-1}(1-\varepsilon) \Omega}\right)$ can be included in the error term $O(\hbar)$, estimate (5.11) yields the following result.

Theorem 5.5. Let Assumption 5.1 hold. Then an arbitrary real solution $\psi(x ; h)$ of equation (1.1) has asymptotics (1.8) for $x \in\left(x_{1}, b_{1}\right)$ and asymptotics (1.9) for $x \in\left(b_{2}, x_{2}\right)$ where $\theta_{j}(\hbar)=$ $\pi / 4(\bmod \pi)$ at least for one $j=1,2$. Formulas (1.8) and (1.9) are uniform in $x$ on compact subintervals of $\left(x_{1}, b_{1}\right)$ and $\left(b_{2}, x_{2}\right)$ and can be differentiated in $x$.

Corollary 5.6. If (1.8) is satisfied with $\theta_{1}(\hbar)=\theta_{1} \neq \pi / 4(\bmod \pi)$, then (1.9) holds true with $\theta_{2}(\hbar)=\pi / 4(\bmod \pi)$. Similarly, if (1.9) is satisfied with $\theta_{2}(\hbar)=\theta_{2} \neq \pi / 4(\bmod \pi)$, then (1.8) holds true with $\theta_{1}(\hbar)=\pi / 4(\bmod \pi)$.

Theorem [5.5 shows that, at least from one side, a potential barrier fixes the phase of the real wave function in the same way as a potential barrier of infinite width (cf. formulas (1.3) and (1.4) where $\left.\varphi_{ \pm}(\hbar)=\pi / 4\right)$. It is convenient to give a formal definition.

Definition 5.7. Let Assumption 5.1 hold, and let $\psi(x ; h)$ be a real solution of equation (1.1) on the interval $\left(x_{1}, x_{2}\right)$. The fixing condition is satisfied at the point $x=b_{j}$ where $j=1$ or $j=2$ if $\theta_{j}(\hbar)=\pi / 4(\bmod \pi)$ in formula (1.8) for $j=1$ or in formula (1.9) for $j=2$.

Remark 5.8. Estimate (5.10) is true for all $\hbar>0$ while the sets of $\hbar$ where (5.11) is satisfied depend on $j=1,2$. This means that there exist sets $\Gamma_{1}, \Gamma_{2} \subset \mathbb{R}_{+}$such that $\Gamma_{1} \cup \Gamma_{2}=\mathbb{R}_{+}$(possibly, $\left.\Gamma_{1} \cap \Gamma_{2} \neq \varnothing\right)$ and $\theta_{j}(\hbar)=\pi / 4(\bmod \pi)$ for $\hbar \in \Gamma_{j}$. 
Remark 5.9. Estimate (5.10) is true for all $\lambda \in\left(\Lambda_{1}, \Lambda_{2}\right)$ while the sets $\Gamma_{j}$ where (5.11) is satisfied may depend on $\lambda$. Note, however, that the derivative of the function (1.7) in $\lambda$ equals

$$
\dot{\Theta}_{j}(x, \lambda)=(-1)^{j} 2^{-1} \int_{b_{j}(\lambda)}^{x}(\lambda-v(y))^{-1 / 2} d y,
$$

and hence it is a bounded function. It follows that

$$
\hbar^{-1}\left|\Theta_{j}\left(x, \lambda^{\prime}\right)-\Theta_{j}\left(x, \lambda^{\prime \prime}\right)\right| \leq C \hbar^{-1}\left|\lambda^{\prime}-\lambda^{\prime \prime}\right|=O(\hbar)
$$

if $\lambda^{\prime}-\lambda^{\prime \prime}=O\left(\hbar^{2}\right)$. Since the phases $\theta_{j}(\hbar)$ in (1.8) and (1.9) are defined up to terms of order $O(\hbar)$, we see that if the fixing condition $\theta_{j}(\hbar)=\pi / 4(\bmod \pi)$ is satisfied for some $j$ and $\lambda$, then it is also satisfied for the same $j$ in a $O\left(\hbar^{2}\right)$ neighborhood of $\lambda$.

5.3. Let us now consider the behavior of the modulus $|\psi(x ; \hbar)|$ as $\hbar \rightarrow 0$ in a neighborhood of a potential barrier. Note that if the fixing condition (5.11) is satisfied at one of the sides of the barrier only, then the solution $\psi(x ; \hbar)$ is localized on the same side. The following statement is a direct consequence of estimates (5.8) or (5.9). As usual, we use equality (5.12).

Theorem 5.10. Under the assumptions of Theorem 5.5 the amplitudes and the phases in (1.8) and (1.9) satisfy the estimate

$$
\frac{A_{k}(\hbar)}{A_{j}(\hbar)} \leq C\left|\sin \vartheta_{k}(\hbar)\right|^{-1} e^{-\hbar^{-1}(1-\varepsilon) \Omega}, \quad j \neq k, \quad \forall \varepsilon>0 .
$$

In particular, if the fixing condition is not satisfied at the point $b_{k}$ (that is, $\left|\sin \vartheta_{k}(\hbar)\right| \geq c>0$ ) then

$$
\frac{A_{k}(\hbar)}{A_{j}(\hbar)} \leq C e^{-\hbar^{-1}(1-\varepsilon) \Omega}, \quad j \neq k, \quad \forall \varepsilon>0 .
$$

Finally, we show that the solution $\psi(x, \hbar)$ decays exponentially inside the potential barrier.

Theorem 5.11. Let the assumptions of Theorem 5.5 be satisfied. Then, for arbitrary $\varepsilon>0$, the estimate

$$
|\psi(x ; \hbar)| \leq C A_{j}(\hbar) e^{\varepsilon \hbar^{-1} \Omega(\lambda)}\left(e^{-\hbar^{-1} \int_{b_{1}}^{x} \sqrt{v(y)-\lambda} d y}+e^{-\hbar^{-1} \int_{x}^{b_{2}} \sqrt{v(y)-\lambda} d y}\right)
$$

holds on all compact subintervals of $\left(b_{1}, b_{2}\right)$ with the same $j$ as in (5.11).

Proof. Let us proceed from formulas (5.1) and (5.2). Suppose, for example, that $j=1$. The estimate

$$
\left|u_{+}\left(x ; b_{1}, \hbar\right)\right| \leq C e^{-\hbar^{-1} \int_{b_{1}}^{x} \sqrt{v(y)-\lambda} d y}
$$

is a direct consequence of (2.12). Combining (2.17) and (5.11) we also see that

$$
\left|w_{+}\left(x ; b_{1}, \hbar\right) \sin \vartheta_{1}(\hbar)\right| \leq C e^{-\hbar^{-1}(1-\varepsilon) \Omega(\lambda)} e^{\hbar^{-1} \int_{b_{1}}^{x} \sqrt{v(y)-\lambda} d y}
$$

which gives the second term in the right-hand side of (5.13). Thus being put together, estimates (5.14) and (5.15) yield (5.13).

Theorem 5.11]is of course a particular case of general S. Agmon's estimates [1] (see also Chapter 6 of the book [6]) on exponential decay of eigenfunctions in a classically forbidden region. 


\section{QuAntization CONDitions FOR MUltiple WELlS}

6.1. Now we are in a position to consider several potential wells. To be precise, we make the following

Assumption 6.1. The function $v \in C^{2}(\mathbb{R})$ and for a point $\lambda_{0} \in \mathbb{R}$, the equation $v(x)=\lambda_{0}$ has only a finite number of solutions, $v^{\prime}(x) \neq 0$ for all $x$ such that $v(x)=\lambda_{0}$ and $v \in C^{3}$ in some neighborhoods of these points. We also suppose that condition (1.10) is satisfied and that function (2.14) is bounded for some $\rho_{0}>1$ as $|x| \rightarrow \infty$.

Let $x_{\ell, \pm}=x_{\ell, \pm}(\lambda), \ell=1, \ldots, L$, be the solutions of the equation $v(x)=\lambda$. We enumerate them as

$$
x_{1,-}<x_{1,+}<x_{2,-}<x_{2,+}<\cdots<x_{L,-}<x_{L,+}
$$

This yields $L$ wells $X_{\ell}(\lambda)=\left(x_{\ell,-}(\lambda), x_{\ell,+}(\lambda)\right), \ell=1, \ldots, L$, separated by $L-1$ potential barriers $B_{\ell}(\lambda)=\left(x_{\ell,+}(\lambda), x_{\ell+1,-}(\lambda)\right), \ell=1, \ldots, L-1$. We also have two infinite barriers $B_{0}(\lambda)=\left(-\infty, x_{1,-}(\lambda)\right)$ and $B_{L}(\lambda)=\left(x_{L,+}(\lambda), \infty\right)$. Of course $\pm v^{\prime}\left(x_{\ell, \pm}(\lambda)\right)>0$ for all $\ell=1, \ldots, L$. For each well, we introduce function (4.1):

$$
\Phi_{\ell}(\lambda)=\int_{x_{\ell,-}(\lambda)}^{x_{\ell,+}(\lambda)}(\lambda-v(x))^{1 / 2} d x .
$$

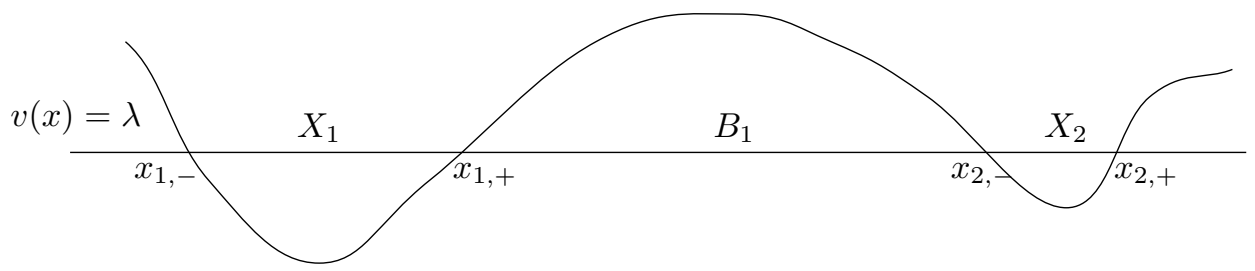

The case $L=2$

Theorem 6.2. Let Assumption 6.1 hold for a point $\lambda_{0}$. Let an eigenvalue $\lambda=\lambda(\hbar)$ of the operator $H(\hbar)$ belong to a neighborhood $\left(\Lambda_{1}, \Lambda_{2}\right)$ of $\lambda_{0}$. Then, at least for one $\ell=\ell(\hbar)=1, \ldots, L$, the estimate

is satisfied, and hence the quantization condition

$$
\cos \left(\hbar^{-1} \Phi_{\ell}(\lambda)\right)=O(\hbar)
$$

$$
\Phi_{\ell}(\lambda)=\pi(n+1 / 2) \hbar+O\left(\hbar^{2}\right)
$$

holds with some integer number $n=n_{\ell}(\hbar)$.

Proof. Theorem 5.5 implies that, for every $\lambda \in\left(\Lambda_{1}, \Lambda_{2}\right)$ and for every $\hbar>0$, each barrier $B_{\ell}(\lambda)$, $\ell=1, \ldots, L-1$, yields at least one fixing condition. Moreover, according to Theorem 2.6 both solutions $u_{ \pm}$of equation (1.1) belong to $L^{2}\left(\mathbb{R}_{ \pm}\right)$. So, if $\lambda$ is an eigenvalue of the operator $H(\hbar)$, then it follows from formula (2.13) that the fixing conditions hold true at the points $x_{1,-}(\lambda)$ and $x_{L,+}(\lambda)$. Thus, for every $\hbar>0$, we have at least $L+1$ fixing conditions for $L$ wells, and hence there exists a well $X_{\ell}(\lambda)$ (depending on $\hbar$ ) for which two fixing conditions are satisfied. Therefore it remains to refer to Theorem 4.4 .

Condition (4.2) on $\dot{\Phi}_{\ell}$ implies that $\Phi_{\ell}$ is a one-to-one mapping of $\left(\Lambda_{1}, \Lambda_{2}\right)$ onto a neighborhood of the point $\mu_{0, \ell}=\Phi_{\ell}\left(\lambda_{0}\right)$. We introduce the inverse mapping $\Psi_{\ell}=\Phi_{\ell}^{-1}:\left(\Phi_{\ell}\left(\Lambda_{1}\right), \Phi_{\ell}\left(\Lambda_{2}\right)\right) \rightarrow$ $\left(\Lambda_{1}, \Lambda_{2}\right)$. Then condition (6.3) for $\lambda=\lambda_{n, \ell}(\hbar)$ is equivalent to the relation

$$
\lambda_{n, \ell}(\hbar)=\Psi_{\ell}(\pi(n+1 / 2) \hbar)+O\left(\hbar^{2}\right) .
$$


Therefore Theorem 6.2 can be reformulated in the following way. Let us consider all intervals

$$
I_{n, \ell}(\hbar)=\left(\Psi_{\ell}(\pi(n+1 / 2) \hbar)-O\left(\hbar^{2}\right), \Psi_{\ell}(\pi(n+1 / 2) \hbar)+O\left(\hbar^{2}\right)\right) \subset\left(\Lambda_{1}, \Lambda_{2}\right) .
$$

Since the lengths of these intervals is of order $\hbar^{2}$ and the distances between the points $\Psi_{\ell}(\pi(n+$ $1 / 2) \hbar)$ and $\Psi_{\ell}(\pi(m+1 / 2) \hbar)$ are of order $\hbar$, we see that $I_{n, \ell}(\hbar) \cap I_{m, \ell}(\hbar)=\varnothing$ if $n \neq m$ and $\hbar$ is sufficiently small. However for different $\ell$, the intersection of two intervals $I_{n, \ell}(\hbar)$ may be non-trivial. Theorem 6.2 means that all eigenvalues of the operator $H(\hbar)$ in the interval $\left(\Lambda_{1}, \Lambda_{2}\right)$ belong to one of the intervals $I_{n, \ell}(\hbar)$, that is,

$$
\operatorname{spec}_{p} H(\hbar) \cap\left(\Lambda_{1}, \Lambda_{2}\right) \subset \cup_{\ell=1}^{L} \cup_{n} I_{n, \ell}(\hbar)
$$

if $\hbar$ is sufficiently small. It can be also equivalently stated as

$$
\operatorname{dist}\left\{\operatorname{spec}_{p} H(\hbar) \cap\left(\Lambda_{1}, \Lambda_{2}\right), \cup_{\ell=1}^{L} \cup_{n} \Psi_{\ell}(\pi(n+1 / 2) \hbar)\right\}=O\left(\hbar^{2}\right) .
$$

To prove the uniqueness of $\lambda$ satisfying (6.3), we need an additional assumption on the remainder $O\left(\hbar^{2}\right)$ in (6.3). The following result is a direct consequence of Proposition 4.6.

Theorem 6.3. Let Assumption 6.1 hold, and let $\lambda$ and $\Phi_{\ell}^{-1}(\pi(n+1 / 2) \hbar)$ belong to a neighborhood of $\lambda_{0}$. Suppose that

$$
\cos \left(\hbar^{-1} \Phi_{\ell}(\lambda)\right)+\epsilon_{\ell}(\lambda, \hbar)=0
$$

where

$$
\epsilon_{\ell}(\lambda, \hbar)=O(\hbar), \quad \dot{\epsilon}_{\ell}(\lambda, \hbar)=o\left(\hbar^{-1}\right) .
$$

Then for every integer $n$, there may exist only one value of $\lambda=\lambda_{n, \ell}(\hbar)$ satisfying condition (6.3).

Being put together, Theorems 6.2 and 6.3 imply that, for a given $\ell$, there is at most one eigenvalue in each of the intervals $I_{n, \ell}(\hbar)$ defined by (6.5).

Let us discuss the converse statement. As usual, we suppose that $\hbar$ is sufficiently small.

Theorem 6.4. Let Assumption 6.1 hold for a point $\lambda_{0}$. Then, for every $\ell=1, \ldots, L$ and every $n$ such that $\Phi_{\ell}^{-1}(\pi(n+1 / 2) \hbar)$ belongs to a neighborhood of $\lambda_{0}$, there exists an eigenvalue $\lambda_{n, \ell}(\hbar)$ of the operator $H(\hbar)$ satisfying relation (6.4).

Proof. Pick some $\ell=1, \ldots, L$ and $n$. We have to construct trial functions $f_{\ell}(x ; \hbar)=f_{n, \ell}(x ; \hbar)$ such that $f_{\ell}(\hbar) \in C_{0}^{2}(\mathbb{R})$ and

$$
\left\|H(\hbar) f_{\ell}(\hbar)-\Psi_{\ell}(\pi(n+1 / 2) \hbar) f_{\ell}(\hbar)\right\| \leq C \hbar^{2}\left\|f_{\ell}(\hbar)\right\|, \quad \forall \hbar>0 .
$$

Let us proceed from Proposition 4.7. For every integer $n$, there exists $\tilde{\lambda}=: \tilde{\lambda}_{n, \ell}(\hbar)$ (so it depends on $\ell, n$ and $\hbar$ ) obeing condition (6.3) such that equality

$$
u_{-}\left(x ; x_{\ell,-}(\tilde{\lambda}), \hbar\right)=a_{\ell}(\tilde{\lambda}, \hbar) u_{+}\left(x ; x_{\ell,+}(\tilde{\lambda}), \hbar\right)
$$

holds true. Moreover, the coefficient $a_{\ell}(\tilde{\lambda}, \hbar)$ satisfies condition (4.18). We fix this value of $\tilde{\lambda}$.

Let a cut-off function $\chi_{\ell} \in C_{0}^{\infty}(\mathbb{R})$ be such that $\chi_{\ell}(x)=1$ in some neighborhood of the interval $X_{\ell}$ and $\chi_{\ell}(x)=0$ away from some larger neighborhood of this interval; in particular, $\chi_{\ell}(x)=0$ on all other intervals $X_{k}, k \neq \ell$. We now set

$$
f_{\ell}(x ; \hbar)=u_{-}\left(x ; x_{\ell,-}(\tilde{\lambda}), \hbar\right) \chi_{\ell}(x) .
$$

Since the function $u_{+}\left(x ; x_{\ell,-}(\tilde{\lambda}), \hbar\right)$ satisfies equation (1.1), we have

$$
\begin{aligned}
-\hbar^{-2} f_{\ell}^{\prime \prime}(x ; \hbar)+(v(x)-\tilde{\lambda}) & f_{\ell}(x ; \hbar) \\
& =-\hbar^{-2}\left(2 u_{-}^{\prime}\left(x ; x_{\ell,-}(\tilde{\lambda}), \hbar\right) \chi_{\ell}^{\prime}(x)+u_{-}\left(x ; x_{\ell,-}(\tilde{\lambda}), \hbar\right) \chi_{\ell}^{\prime \prime}(x)\right) .
\end{aligned}
$$


Due to the functions $\chi_{\ell}^{\prime}$ and $\chi_{\ell}^{\prime \prime}$, this expression differs from zero on compact subsets of the barriers $B_{\ell-1}$ and $B_{\ell}$ only. Relation (2.12) shows that expression (6.11) tends to zero exponentially as $\hbar \rightarrow 0$ on the left barrier $B_{\ell-1}$. In view of (6.10), we can rewrite (6.11) as

$$
-a_{\ell}(\tilde{\lambda}, \hbar) \hbar^{-2}\left(2 u_{+}^{\prime}\left(x ; x_{\ell,+}(\tilde{\lambda}), \hbar\right) \chi_{\ell}^{\prime}(x)+u_{+}\left(x ; x_{\ell,+}(\tilde{\lambda}), \hbar\right) \chi_{\ell}^{\prime \prime}(x)\right),
$$

and so according to relation (4.18) for $a_{\ell}(\tilde{\lambda}, \hbar)$, it also tends to zero exponentially as $\hbar \rightarrow 0$ on the right barrier $B_{\ell}$. Therefore (6.11) implies that

$$
\left\|H(\hbar) f_{\ell}(\hbar)-\tilde{\lambda}(\hbar) f_{\ell}(\hbar)\right\| \leq C \hbar^{p}\left\|f_{\ell}(\hbar)\right\|, \quad \forall p>0 .
$$

To conclude the proof of the inequality (6.9), it remains to use that

$$
\tilde{\lambda}(\hbar)-\Psi_{\ell}(\pi(n+1 / 2) \hbar)=O\left(\hbar^{2}\right)
$$

according to condition (6.3) on $\tilde{\lambda}(\hbar)$.

Remark 6.5. The support of the trial function $f_{\ell}(x ; \hbar)$ lies in a small neighborhood of the interval $X_{\ell}$. Therefore, for different $\ell$, the supports of $f_{\ell}(x ; \hbar)$ are disjoint.

6.2. Here we discuss some consequences of the results obtained. Let $N(\hbar)$ be the total number of eigenvalues of the operator $H(\hbar)$ in the interval $\left(\Lambda_{1}, \Lambda_{2}\right)$. For each $\ell=1, \ldots, L$, we consider the intervals $I_{n, \ell}(\hbar)$ defined by formula (6.5). Since $I_{n, \ell}(\hbar) \cap I_{m, \ell}(\hbar)=\varnothing$ if $n \neq m$ and $\hbar$ is sufficiently small, the total number $\mathcal{N}_{\ell}(\hbar)$ of such intervals lying in $\left(\Lambda_{1}, \Lambda_{2}\right)$ obeys a two-sided bound

$$
\left(\Phi_{\ell}\left(\Lambda_{2}\right)-\Phi_{\ell}\left(\Lambda_{1}\right)\right)(\pi \hbar)^{-1}-1 \leq \mathcal{N}_{\ell}(\hbar) \leq\left(\Phi_{\ell}\left(\Lambda_{2}\right)-\Phi_{\ell}\left(\Lambda_{1}\right)\right)(\pi \hbar)^{-1}+1 .
$$

Proposition 6.6. Let Assumption 6.1 hold for a point $\lambda_{0}$. Then

$$
N(\hbar) \geq \sum_{\ell=1}^{L}\left(\Phi_{\ell}\left(\Lambda_{2}\right)-\Phi_{\ell}\left(\Lambda_{1}\right)\right)(\pi \hbar)^{-1}-L
$$

for sufficiently small $\hbar$.

Proof. According to Theorem 6.4. for every $\ell=1, \ldots, L$ and $n$, there is an eigenvalue of the operator $H(\hbar)$ lying in each interval $I_{n, \ell}(\hbar)$. Although for different $\ell$, the intersection of the intervals $I_{n, \ell}(\hbar)$ may be non-trivial, the corresponding trial functions have disjoint supports (see Remark 6.5). It follows that

$$
N(\hbar) \geq \sum_{\ell=1}^{L} \mathcal{N}_{\ell}(\hbar) .
$$

So, it suffices to use the left estimate (6.12).

We emphasize that the lower bound (6.13) does not require the results of Section 5 on a passage through a potential barrier.

Proposition 6.7. Let the assumptions of Theorem 6.3 be satisfied for all $\ell=1, \ldots, L$. Then

$$
N(\hbar) \leq \sum_{\ell=1}^{L}\left(\Phi_{\ell}\left(\Lambda_{2}\right)-\Phi_{\ell}\left(\Lambda_{1}\right)\right)(\pi \hbar)^{-1}+L
$$

if $\hbar$ is sufficiently small $\hbar$. 
Proof. Using inclusion (6.6) and the fact that there is at most one eigenvalue in each of the intervals $I_{n, \ell}(\hbar)$, we find that

$$
N(\hbar) \leq \sum_{\ell=1}^{L} \mathcal{N}_{\ell}(\hbar) .
$$

So, it suffices to use the right estimate (6.12).

In view of definition (6.3) of the function $\Phi_{\ell}$ we have

$$
\begin{aligned}
\sum_{\ell=1}^{L}\left(\Phi_{\ell}\left(\Lambda_{2}\right)-\Phi_{\ell}\left(\Lambda_{1}\right)\right) & =2^{-1} \iint_{\Lambda_{1}<p^{2}+v(x)<\Lambda_{2}} d p d x \\
& =2^{-1} \operatorname{mes}\left\{(x, p) \in \mathbb{R}^{2}: \Lambda_{1}<p^{2}+v(x)<\Lambda_{2}\right\} .
\end{aligned}
$$

Thus, estimates (6.13) and (6.14) yield the semiclassical Weyl formula with a strong estimate of the remainder. Unfortunately the upper bound (6.14) requires implicit assumption (6.8).

6.3. Let us discuss the case $L=2$ in some details. For short, we set $u_{-}=u_{-}\left(x_{1,-}\right), u_{+}=$ $u_{+}\left(x_{2,+}\right), u_{1}=u_{+}\left(x_{1,+}\right), w_{1}=w_{+}\left(x_{1,+}\right)$ and $u_{2}=u_{-}\left(x_{2,-}\right), w_{2}=w_{-}\left(x_{2,-}\right)$. Let $\lambda=\lambda(\hbar)$ be an eigenvalue and $\psi(x)=\psi(x ; \lambda, \hbar)$ be a real eigenfunction of the operator $H(\hbar)$. Since $u_{ \pm} \in L^{2}\left(\mathbb{R}_{ \pm}\right)$ and the functions $u_{\ell}, w_{\ell}$ are linearly independent, we have

$$
\psi=A_{\ell}\left(\cos \phi_{\ell} u_{\ell}+\sin \phi_{\ell} w_{\ell}\right), \quad x \in\left(x_{\ell,-}, x_{\ell,+}\right), \quad \ell=1,2 .
$$

On the other hand, $\psi=A_{ \pm} u_{ \pm}$because $u_{ \pm} \in L^{2}\left(\mathbb{R}_{ \pm}\right)$. It follows that

$$
\gamma_{1}:=\tan \phi_{1}=-\frac{\left\{u_{-}, u_{1}\right\}}{\left\{u_{-}, w_{1}\right\}}, \quad \gamma_{2}:=\tan \phi_{2}=-\frac{\left\{u_{+}, u_{2}\right\}}{\left\{u_{+}, w_{2}\right\}} .
$$

The condition

$$
\left\{u_{1}+\gamma_{1} w_{1}, u_{2}+\gamma_{2} w_{2}\right\}=0
$$

yields the equation

$$
\left(\gamma_{1}+\frac{\left\{u_{1}, w_{2}\right\}}{\left\{w_{1}, w_{2}\right\}}\right)\left(\gamma_{2}+\frac{\left\{w_{1}, u_{2}\right\}}{\left\{w_{1}, w_{2}\right\}}\right)=-\left\{u_{1}, u_{2}\right\}+\frac{\left\{u_{1}, w_{2}\right\}\left\{w_{1}, u_{2}\right\}}{\left\{w_{1}, w_{2}\right\}^{2}}=: \omega_{0}
$$

for $\gamma_{1}$ and $\gamma_{2}$.

According to Lemma 5.2 we have

$$
\omega_{1}:=\frac{\left\{u_{1}, w_{2}\right\}}{\left\{w_{1}, w_{2}\right\}}=O\left(e^{-\hbar^{-1}(2-\varepsilon) \Omega}\right), \quad \omega_{2}:=\frac{\left\{w_{1}, u_{2}\right\}}{\left\{w_{1}, w_{2}\right\}}=O\left(e^{-\hbar^{-1}(2-\varepsilon) \Omega}\right)
$$

for all $\varepsilon>0$ and

$$
\omega_{0}=2^{-1} \hbar^{-1} e^{-\hbar^{-1} \Omega}(1+O(\hbar))
$$

Moreover, these relations may be differentiated in $\lambda$. It follows from Lemma 4.2 that numbers (6.15) are given by the formula

$$
\gamma_{\ell}(\hbar)=\frac{\cos \left(\hbar^{-1} \Phi_{\ell}\right)+\sigma_{\ell}(\hbar)}{\sin \left(\hbar^{-1} \Phi_{\ell}\right)+\eta_{\ell}(\hbar)}
$$

where the functions $\Phi_{\ell}$ are defined by (6.1) and

$$
\sigma_{\ell}(\hbar)=O(\hbar), \quad \eta_{\ell}(\hbar)=O(\hbar), \quad \dot{\sigma}_{\ell}(\hbar)=O(1), \quad \dot{\eta}_{\ell}(\hbar)=O(1)
$$

as $\hbar \rightarrow 0$.

In view of (6.17), (6.18), equation (6.16) implies that

$$
\min \left\{\left|\gamma_{1}(\hbar)+\omega_{1}(\hbar)\right|,\left|\gamma_{2}(\hbar)+\omega_{2}(\hbar)\right|\right\} \leq \hbar^{-1 / 2} e^{-(2 \hbar)^{-1} \Omega} .
$$


Suppose, for example, that the first term on the left is smaller than the second one. Then the conditions (6.2) and (6.3) for $\ell=1$ follow from estimates (6.17) and (6.21).

We will now discuss the second condition (6.8) for $\ell=1$. To that end, we need an explicit expression for the remainder $\epsilon_{1}(\hbar)$ in (6.7). It follows from (6.16) and (6.19) that equation (6.7) for $\ell=1$ is satisfied with

$$
\epsilon_{1}(\hbar)=\sigma_{1}(\hbar)+\left(\sin \left(\hbar^{-1} \Phi_{1}\right)+\eta_{1}(\hbar)\right)\left(\omega_{1}(\hbar)-\frac{\omega_{0}(\hbar)}{\gamma_{2}(\hbar)+\omega_{2}(\hbar)}\right)
$$

where of course all terms depend on $\lambda$. Let us differentiate this equality in $\lambda$. According to relations (6.17), (6.18) and (6.20) for the proof of the second estimate (6.8), we only have to exclude that the denominator $\gamma_{2}(\hbar)+\omega_{2}(\hbar)$ tends to zero exponentially as $\hbar \rightarrow 0$. In view of (6.19) we have

$$
\frac{1}{\gamma_{2}(\hbar)+\omega_{2}(\hbar)}=\frac{\sin \left(\hbar^{-1} \Phi_{2}\right)+\eta_{2}(\hbar)}{\cos \left(\hbar^{-1} \Phi_{2}\right)+\sigma_{2}(\hbar)+\omega_{2}(\hbar)\left(\sin \left(\hbar^{-1} \Phi_{2}\right)+\eta_{2}(\hbar)\right)} .
$$

This expression is $O\left(\hbar^{-1}\right)$ provided $\left|\cos \left(\hbar^{-1} \Phi_{2}\right)\right| \geq c \hbar$ for a sufficiently large $c>0$. Roughly speaking, this means that the quantization condition is not satisfied in the well $X_{2}$. Thus Theorem 6.3 can be supplemented by the following assertion. Recall that the intervals $I_{n, \ell}(\hbar)$ were defined by relation (6.5).

Proposition 6.8. Let Assumption 6.1 hold, and let $L=2$. Then the operator $H(\hbar)$ has at most one eigenvalue in an interval $I_{n, \ell}(\hbar)$ provided

$$
\left|\cos \left(\hbar^{-1} \Phi_{k}(\lambda)\right)\right| \geq c \hbar, \quad k \neq \ell, \quad \lambda \in I_{n, \ell}(\hbar),
$$

for a sufficiently large $c>0$.

So, the problems with estimate (6.8) may arise if the quantization conditions (6.2) are satisfied in both wells. However this estimate may still be true under both quantization conditions. As an example, consider the symmetric double well when $L=2$ and $v(-x)=v(x)$. Then $\Phi_{1}=\Phi_{2}=: \Phi$, $\gamma_{1}=\gamma_{2}=: \gamma, \sigma_{1}=\sigma_{2}=: \sigma, \eta_{1}=\eta_{2}=: \eta$ and $\omega_{1}=\omega_{2}=: \omega$. In this case it follows from (6.16) that

$$
\gamma=-\omega \pm \sqrt{\omega_{0}}
$$

which yields 2 equations (6.7) for $\Phi$ with

$$
\epsilon_{\ell}(\hbar)=\sigma(\hbar)+\left(\sin \left(\hbar^{-1} \Phi\right)+\eta(\hbar)\right)\left(\omega+(-1)^{\ell} \sqrt{\omega_{0}}\right), \quad \ell=1,2 .
$$

These functions satisfy the estimates $\epsilon_{\ell}(\hbar)=O(\hbar)$ and $\dot{\epsilon}_{\ell}(\hbar)=O(1)$, and hence conditions (6.8) are true for both $\ell$. Thus there is exactly one eigenvalue of the operator $H(\hbar)$ in each interval $I_{n, \ell}(\hbar), \ell=1,2$. Note also that since the second term in the right-hand side of (6.22) decays exponentially, the intervals $I_{n, 1}(\hbar)$ and $I_{n, 2}(\hbar)$ defined up to the length $O\left(\hbar^{2}\right)$ may be identified. Then one can say that there are exactly 2 eigenvalues in each interval $I_{n, 1}(\hbar)=I_{n, 2}(\hbar)$.

\section{Appendix A. Tunneling of a PARTicle through a Potential Barrier}

Here we again consider a potential barrier and accept Assumption [5.1. In contrast to bound states, tunneling of a particle (wave) through a barrier is described by complex solutions of equation (1.1). They are again distinguished by their asymptotics away from the barrier. Similarly to Section 5 , the energy $\lambda$ is fixed.

Let us consider the solutions

$$
\begin{aligned}
& f_{1}(x ; \hbar)=i w_{+}\left(x ; b_{1}, \hbar\right)+u_{+}\left(x ; b_{1}, \hbar\right) \\
& f_{2}(x ; \hbar)=w_{-}\left(x ; b_{2}, \hbar\right)+i u_{-}\left(x ; b_{2}, \hbar\right)
\end{aligned}
$$


of equation (1.1). In view of relations (2.13) and (2.18) they have the asymptotics

$$
f_{j}(x ; \hbar)=(\lambda-v(x))^{-1 / 4} \exp \left(i \hbar^{-1} \int_{b_{j}}^{x}(\lambda-v(y))^{1 / 2} d y+\pi i / 4\right)+O(\hbar)
$$

as $\hbar \rightarrow 0$ on compact subsets of the interval $\left(x_{1}, b_{1}\right)$ for $f_{1}(x ; \hbar)$ and of the interval $\left(b_{2}, x_{2}\right)$ for $f_{2}(x ; \hbar)$. The function $f_{1}(x ; \hbar)$ corresponds to the incident wave propagating from $x=-\infty$ in the direction of the barrier. Its part reflected by the barrier is described by the function $\overline{f_{1}(x ; \hbar)}$, and the part transmitted through the barrier is described by the function $f_{2}(x ; \hbar)$.

It follows from formula (2.19) that the Wronskian

$$
\left\{f_{1}(\hbar), \overline{f_{1}(\hbar)}\right\}=-2 i\left\{u_{+}\left(b_{1}, \hbar\right), w_{+}\left(b_{1}, \hbar\right)\right\}=2 i \hbar^{-1}(1+O(\hbar)),
$$

so that the functions $f_{1}(x ; \hbar)$ and $\overline{f_{1}(x ; \hbar)}$ are linearly independent and hence

$$
f_{2}(x ; \hbar)=A(\hbar) f_{1}(x ; \hbar)+B(\hbar) \overline{f_{1}(x ; \hbar)}
$$

where the complex numbers $A(\hbar)$ and $B(\hbar)$ are determined by the equations

$$
\left\{f_{1}(\hbar), \overline{f_{1}(\hbar)}\right\} A(\hbar)=\left\{f_{2}(\hbar), \overline{f_{1}(\hbar)}\right\}, \quad\left\{f_{1}(\hbar), \overline{f_{1}(\hbar)}\right\} B(\hbar)=\left\{f_{1}(\hbar), f_{2}(\hbar)\right\} .
$$

Recall that the function $\Omega$ is defined by formula (5.3). According to Lemma 5.2 we have

$$
\begin{aligned}
\left\{f_{2}(\hbar), \overline{f_{1}(\hbar)}\right\} & =\left\{w_{-}\left(b_{2}, \hbar\right)+i u_{-}\left(b_{2}, \hbar\right),-i w_{+}\left(b_{1}, \hbar\right)+u_{+}\left(b_{1}, \hbar\right)\right\} \\
& =2 i \hbar^{-1} e^{\hbar^{-1} \Omega}(1+O(\hbar))
\end{aligned}
$$

and, similarly,

$$
\begin{aligned}
\left\{f_{2}(\hbar), f_{1}(\hbar)\right\} & =\left\{w_{-}\left(b_{2}, \hbar\right)+i u_{-}\left(b_{2}, \hbar\right), i w_{+}\left(b_{1}, \hbar\right)+u_{+}\left(b_{1}, \hbar\right)\right\} \\
& =-2 i \hbar^{-1} e^{\hbar^{-1} \Omega}(1+O(\hbar)) .
\end{aligned}
$$

Substituting (A.3), (A.6) and (A.7) into equations (A.5), we find asymptotic expressions for $A(\hbar)$ and $B(\hbar)$ :

$$
A(\hbar)=e^{\hbar^{-1} \Omega}(1+O(\hbar)), \quad B(\hbar)=e^{\hbar^{-1} \Omega}(1+O(\hbar)) .
$$

Dividing (A.4) by $A(\hbar)$, we see that

$$
f_{1}(x ; \hbar)+R(\hbar) \overline{f_{1}(x ; \hbar)}=T(\hbar) f_{2}(x ; \hbar)
$$

where $R(\hbar)=A(\hbar)^{-1} B(\hbar)$ and $T(\hbar)=A(\hbar)^{-1}$ are known as the reflection and transmission coefficients for scattering of the wave $f_{1}(x ; \hbar)$ by the potential barrier $v(x)$. It follows from formulas (A.8) that

$$
R(\hbar)=1+O(\hbar) \quad \text { and } \quad T(\hbar)=e^{-\hbar^{-1} \Omega}(1+O(\hbar))
$$

Thus we have obtained the following result.

Theorem A.1. Let the solutions $f_{1}(x ; \hbar)$ and $f_{2}(x ; \hbar)$ of equation (1.1) be defined by formula (A.1). They have asymptotics (A.2) as $\hbar \rightarrow 0$ and are linked by equality (A.9). The asymptotics as $\hbar \rightarrow 0$ of the reflection $R(\hbar)$ and of the transmission $T(\hbar)$ coefficients are given by formulas (A.10). 


\section{REFERENCES}

[1] Agmon S., Lectures on exponential decay of solutions of second order elliptic equations, Math. Notes of Princeton Univ., 29, 1982.

[2] Buslaev V. S., Semiclassical approximation for equations with periodic coefficients, Russ. Math. Surveys 42 (1987), 97-125.

[3] Buslaev V. S., Dmitrieva L. A., A Bloch electron in an external field, Leningrad Math. J. 1 no. 2, (1990), 287-320.

[4] Buslaev V., Grigis A., Turning points for adiabatically perturbed periodic equations, J. Anal. Math. 84 (2001), $67-143$.

[5] Colin de Verdière I., Parisse B., Singular Bohr-Sommerfeld rules, Comm. Math. Phys. 205 (1999), 459-500.

[6] Dimassi M., Sjöstrand J., Spectral asymptotics in the semi-classical limit, LMS Lecture Notes Series 268, Cambridge Univ. Press, 1999.

[7] Fedoryuk M. V., Asymptotic methods for linear ordinary differential equations, Nauka, 1983 (Russian).

[8] Ford K. W., Hill D. L., Wakeno M., Wheeler J. A., Quantum effect near a barrier maximum, Ann. Physics 7 (1959), 239-258.

[9] Harrel E. M., On the rate of asymptotic eigenvalue degeneracy, Comm. Math. Phys. 60 (1978), 73-95.

[10] Harrel E. M., Double wells, Comm. Math. Phys. 75 (1980), 239-261.

[11] Helffer B., Robert D., Puits de potentiels généralisés, Ann. Institut H. Poincaré, phys. théor., 41, no 3, (1984), 291-331.

[12] Helffer B., Shöstrand J., Multiple wells in the semi-classical limit. I, Comm. Part. Diff. Eq. 9 (1984), 337-408.

[13] Jeffreys H., On certain approximate solutions of linear differential equations of the second order, Proc. London Math. Soc. [2] 23 (1924), 428-436.

[14] Kirsch W., Simon, B., Universal lower bounds on eigenvalue splittings for one dimensional Schrödinger operators, Comm. Math. Phys. 97 (1985), 453-460.

[15] Kramers H. A., Wellenmechanik und halbzahlige Quantisiering, Zeitschrift für Physik. 39 (1926), 828-840.

[16] Landau L. D., Lifshitz E. M., Classical mechanics, Pergamon Press, 1960.

[17] Landau L. D., Lifshitz E. M., Quantum mechanics, Pergamon Press, 1965.

[18] Langer R. E., On the connection formulas and the solutions of the wave equation, Phys. Rev., 51 (1937), 669-676.

[19] Langer R. E., The asymptotic solutions of ordinary linear differential equations of the second order, with a special reference to a turning point, Trans. Amer. Math. Soc. 67 (1949), 461-490.

[20] Nakamura S., A remark on eigenvalue splittings for one-dimensional double-well Hamiltonians, Lett. Math. Phys. 11 (1986), 337-340.

[21] Olver F. W. J., Asymptotics and special functions, Academic Press, 1974.

[22] Robert D., Autour de l'approximation semi-classique, Progress in Math. 68, Birkhäuser, 1987.

[23] Simon B., Semiclassical analysis of low lying eigenvalues, II, Tunneling, Annals of Math. 120, (1984), 89-118.

[24] Titchmarsh E. C., Eigenfunction expansions associated with second-order differential equations, vol. 1, Oxford, 1946.

[25] Yafaev D. R., The semiclassical limit of eigenfunctions of the Schrödinger equation and the Bohr-Sommerfeld quantization condition, revisited, St.-Petersburg Math. J. 22 no. 6, (2011), 1051-1067.

irmar, Université de Rennes I, Campus de Beaulieu, Rennes, 35042 France and SPGU, Univ. Nab. 7/9, Saint Petersburg, 199034 RUSSiA

E-mail address: yafaev@univ-rennes1.fr 